\title{
A CMOS-Compatible Spectrum Analyzer for Cognitive Radio Exploiting Crosscorrelation to Improve Linearity and Noise Performance
}

\author{
Mark S. Oude Alink, Student Member, IEEE, Eric A.M. Klumperink, Senior Member, IEEE, André B.J. Kokkeler, \\ Michiel C.M. Soer, Student Member, IEEE, Gerard J.M. Smit, Bram Nauta, Fellow, IEEE
}

\begin{abstract}
A spectrum analyzer requires a high linearity to handle strong signals, and at the same time a low NF to enable detection of much weaker signals. This is not only important for lab equipment, but also for the spectrum sensing part of cognitive radio, where low cost and integration is at a premium. Often there is a trade-off between linearity and noise: improving one degrades the other. Crosscorrelation can break this tradeoff by reducing noise at the expense of measurement time. An existing RF frontend in CMOS-technology with IIP3=+11 dBm and $\mathrm{NF}=5.5 \mathrm{~dB}$ is duplicated and attenuators are put in front to increase linearity to IIP3 $=+24 \mathrm{dBm}$. The attenuation degrades NF, but by using crosscorrelation of the outputs of the two frontends, the effective NF is reduced to around $5 \mathrm{~dB}$. In total, this results in a spurious-free dynamic range of $88 \mathrm{~dB}$ in $1 \mathrm{MHz}$ resolution bandwidth.
\end{abstract}

Index Terms-cognitive radio, crosscorrelation, energy detection, IIP3, linearity, noise figure, phase noise, power detection, spectrum analyzer, spectrum sensing, spurious-free dynamic range

\section{INTRODUCTION}

$\mathbf{S}$ PECTRUM SENSING is one of the key features that defines cognitive radio (CR): a new paradigm targeting more efficient use of the spectrum by opportunistically using available frequencies, instead of the static frequency assignment that is in common use today. A CR-implementation may benefit from a spectrum analyzer (SA) of some form to find unoccupied bandwidth (also known as "spectrum hole" [1], "white space" [2], or "white spot" [3]). In contrast with lab equipment, where power consumption and costs are not a primary concern, a SA for CR should be low-cost, compact, and low-power. Because CRs will also require a fair share of digital signal processing (DSP), CMOS-technology seems like the ideal candidate. However, CMOS has its limitations, especially in terms of linearity due to the low supply voltage.

Manuscript received February 2, 2011; revised June 1, 2011 and July 20, 2011.

Copyright (c) 2011 IEEE. Personal use of this material is permitted. However, permission to use this material for any other purposes must be obtained from the IEEE by sending a request to pubs-permissions@ieee.org.

M. S. Oude Alink is with the Computer Architecture for Embedded Systems (CAES) Group and the IC-Design Group, Centre for Telematics and Information Technology (CTIT), University of Twente, 7500 AE Enschede, The Netherlands (e-mail: m.s.oudealink@utwente.nl).

A. B. J. Kokkeler and G. J. M. Smit are with the CAES Group, CTIT, University of Twente, $7500 \mathrm{AE}$ Enschede, The Netherlands.

E. A. M. Klumperink, M. C. M. Soer and B. Nauta are with the IC-Design Group, CTIT, University of Twente, 7500 AE Enschede, The Netherlands.
It is shown in [3] that, depending on the spectral location of strong signals, each white spot poses different linearity requirements, some of which cannot be met with state-of-the-art receivers. These spots, e.g. where third-order intermodulation (IM3) distortion occurs, can be identified with a SA [3], [4], but to avoid false alarms, the SA should not suffer itself from the distortion. Attenuation of the input signal lowers the distortion components, but raises the noise floor, which obscures weak signals and therefore causes missed detections.

This noise-linearity trade-off is captured in the spurious-free dynamic range (SFDR), which is defined as the difference in decibels between the strongest and weakest signal that can be detected at the same time [5]. The importance of a large SFDR is stressed by the observation of the Federal Communications Commission (FCC) that industrial prototypes were able to detect very weak signals in an otherwise clean spectrum, but failed in the presence of a large interferer [2]. The SFDR is limited by non-linearity (IIP2, IIP3) and noise (both thermal and phase noise) [6]. Definitions of SFDR differ between fields (e.g., for analog-to-digital converters (ADCs), only the nonlinearity is taken into account, and not the noise) and even between authors in the same field (e.g., for receivers, Razavi [7] includes minimum SNR for demodulation, while Lee [5] does not). We assume the definition as used for the SFDR in SA-datasheets, where it is taken that distortion and noise floor are at the same level. The following equation for the SFDR is valid if it is limited by NF and IIP3 [5], [6]

$$
\mathrm{SFDR}=\frac{2}{3}\left(\mathrm{IIP} 3-\mathrm{NF}-10 \log _{10} B+174\right)[\mathrm{dB}]
$$

where $B$ is the resolution bandwidth (RBW).

In this paper we will demonstrate that it is possible to improve both linearity and noise of a SA, and hence SFDR, by using two identical RF-frontends in combination with attenuators and crosscorrelation. At the expense of increased measurement time, compared to the single RF-frontend [8], a $13 \mathrm{~dB}$ higher IIP3 can be obtained without compromising noise performance, increasing SFDR by $9 \mathrm{~dB}$. Part of this paper is also presented in [9], but here we add 1) an analysis of impedance matching and measurements thereof, 2) the principle of phase noise reduction and measurements thereof, 3) a discussion on digital correlator architectures, and 4) comparison of calculations of NF (before, during, and after correlation) and IIP3 with new measurements.

Section II briefly explains the basics of crosscorrelation 


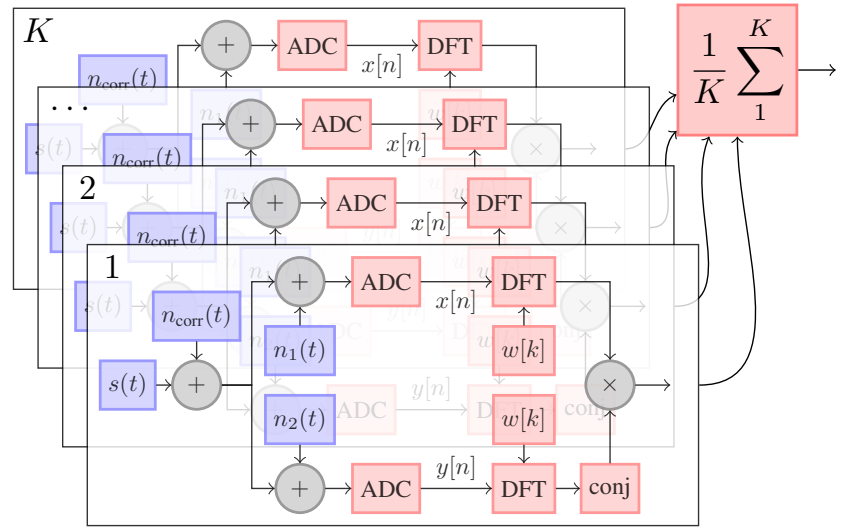

Fig. 1. The FXC first calculates the (windowed) Fourier transforms of the incoming samples to arrive at a cross-spectrum, and then averages several cross-spectra to lower the variance.

and its DSP-implementation. Section III discusses the implementation of our crosscorrelation spectrum analyzer (XCSA), followed by an analysis of its expected performance in section IV and measurement results in section V. We end with conclusions in section VI.

\section{CROSSCORRELATION}

Since crosscorrelation is such an important topic in our design, we will first briefly consider its mathematical background in section II-A and its DSP-implementation in section II-B. We will continue with the important relation between crosscorrelation and NF in section II-C, followed by a discussion on the sensitivity limit of crosscorrelation in section II-D. We wrap up this section with an analysis on the impact of receiver mismatch in section II-E.

\section{A. Mathematical Background}

The Wiener-Khinchin theorem states that the power spectrum $\Gamma_{X X}(f)$ and the autocorrelation function (acf) $\gamma_{X X}(\tau)$ form a Fourier transform pair [10]:

$$
\begin{aligned}
& \Gamma_{X X}(f)=\mathcal{F}\left(\gamma_{X X}(\tau)\right)=\int_{-\infty}^{\infty} \gamma_{X X}(\tau) e^{-j 2 \pi f \tau} \mathrm{d} \tau \\
& \gamma_{X X}(\tau)=\mathcal{F}^{-1}\left(\Gamma_{X X}(f)\right)=\int_{-\infty}^{\infty} \Gamma_{X X}(f) e^{j 2 \pi f \tau} \mathrm{d} f
\end{aligned}
$$

with the acf defined as

$$
\gamma_{X X}(\tau) \triangleq \mathbb{E}\left[X(t) X^{*}(t+\tau)\right] .
$$

Assuming ergodic processes, the time average of one realization equals the ensemble average

$$
\gamma_{X X}(\tau)=\lim _{T \rightarrow \infty} \frac{1}{2 T} \int_{-T}^{T} x(t) x^{*}(t+\tau) \mathrm{d} t
$$

where $T$ is the measurement time. This means that an estimate of the acf can be made by observing one realization for a certain amount of time. Since the observation time is limited, the Fourier transform can only be approximated. To reduce the spectral leakage that is caused by the convolution with the finite time window, windowing can be used.

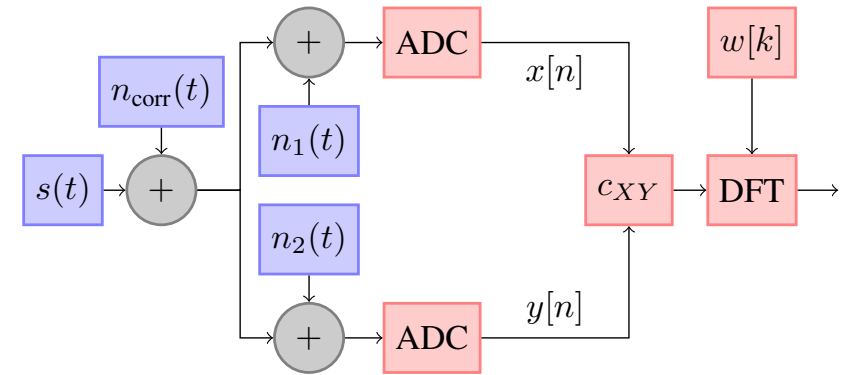

(a) Block scheme of XFC

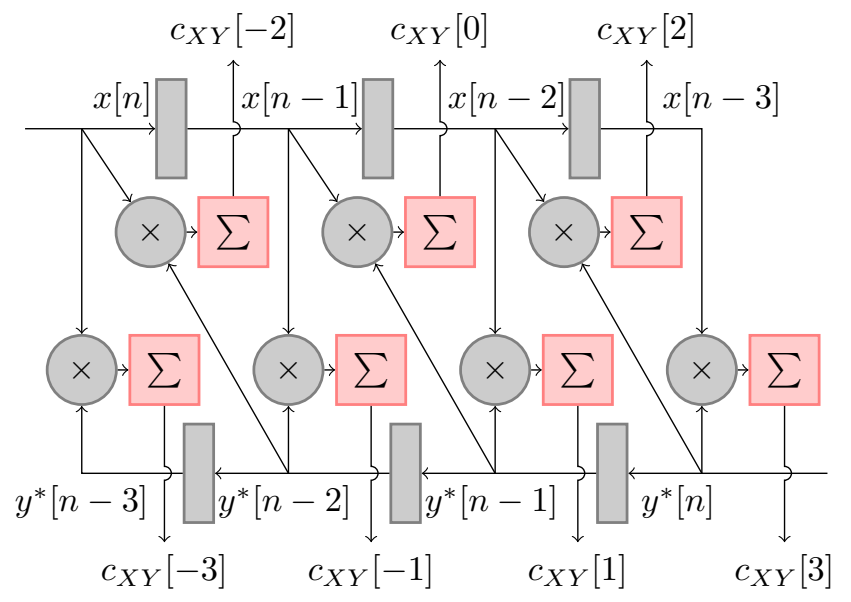

(b) Implementation of $c_{X Y}$

Fig. 2. The XFC first estimates the ccf of the incoming samples to arrive at the cross-spectrum estimate.

The same derivations hold for the crosscorrelation function (ccf), defined as

$$
\gamma_{X Y}(\tau) \triangleq \mathbb{E}\left[X(t) Y^{*}(t+\tau)\right]
$$

Fourier-transforming the ccf instead of the acf results in a so-called cross power spectrum (or simply cross-spectrum) $\Gamma_{X Y}(f)$, which in general is a complex function of frequency.

\section{B. Digital correlators}

Crosscorrelation can be performed in the analog or the digital domain, but since it is desirable to reuse the receiver as part of the SA, and in CMOS digital circuits scale much better than analog circuits, we only consider digital correlators. The two main algorithms for digital correlation are known as the FX-correlator (FXC) and the XF-correlator (XFC) [11], which differ in the order in which crosscorrelation $(\mathrm{X})$ and the Fourier transform (F) (using the fast Fourier transform (FFT) for efficiency) are calculated.

The principle of the FXC is shown in Fig. 1, where the cross-spectrum estimate $C[f]$ is obtained by Fouriertransforming the receiver outputs $x[n]$ and $y[n]$ to obtain $X[f]$ and $Y[f]$, and then calculating $C[f]=X[f] Y^{*}[f]$. To reduce the variance, the calculations are repeated with new samples and the results are averaged.

The XFC estimates the cross-spectrum by first estimating the ccf $\gamma_{X Y}(\tau)$, denoted by $c_{X Y}[k]$, and then taking the discrete Fourier transform (DFT) thereof to arrive at $C[f]$, 


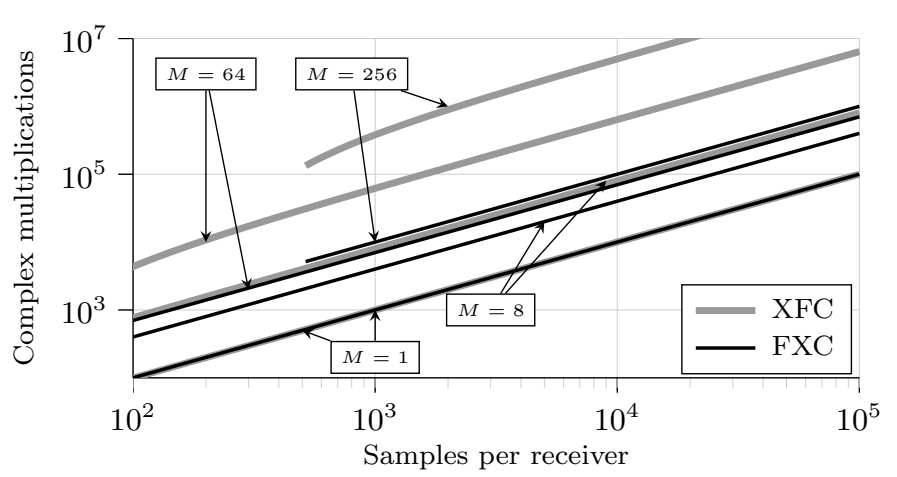

Fig. 3. The number of complex multiplications for the FXC and the XFC for an $M$-point spectral estimate.

see Fig. 2. For an $M$-point spectral estimate, $M$ points in the ccf need to be calculated.

An $M$-point FFT in combination with a sample rate $f_{s}$ gives a RBW of $f_{s} / M$. For spectral estimation, there is a trade-off between RBW and measurement time: the variance of each sample at the output of an FFT is independent of $M$, so a 2 times narrower RBW requires a 2 times longer measurement time to obtain the same variance in each bin. This is true for crosscorrelation as well. Moreover, a larger $M$ also brings along with it a higher computational complexity: the basic Cooley-Tukey algorithm requires $\frac{M}{2} \log _{2} M$ complex multiplications and $M \log _{2} M$ complex additions. The computational burden (showing only complex multiplications) is depicted in Fig. 3 for the FXC and XFC for an $M$-point spectral estimate. For $M=1$, both reduce to a single multiplyaccumulate (MAC)-unit.

Even though the complexity largely favors the FXC, there are some differences in performance with respect to wordlength and a small loss in SNR, which has made the XFC the most popular in radio-astronomy [11]. In our implementation, the processing takes place on a general purpose processor (GPP), which makes the FXC by far the most attractive option due to its lower computational complexity. Moreover, orthogonal frequency division multiplexing (OFDM) is often mentioned in the CR-context as the modulation to use, due to its flexibility in bandwidth. Since OFDM extensively uses FFTs for modulation and demodulation, it is likely that an efficient implementation of FFTs will be available in CRs.

\section{Crosscorrelation and NF}

The frontend of the SA adds noise to the signal, which, due to the autocorrelation (energy detection) that is performed, results in the power spectral density (PSD) of the signal plus noise, so small signals will be obscured by the noise.

We use the system model of Fig. 4 to explain the principle of crosscorrelation in terms of noise reduction. The signal $s(\mathrm{t})$ is assumed independent of the noise, and the noise is modeled as three independent parts: $n_{\text {corr }}$ which is correlated in both frontends, and $n_{1}(\mathrm{t})$ and $n_{2}(\mathrm{t})$ that are only present in one of the individual frontends. We define $r_{1}(\mathrm{t})$ and $r_{2}(\mathrm{t})$ as the output signals of the individual receivers, and $q(\mathrm{t})$ as the sum

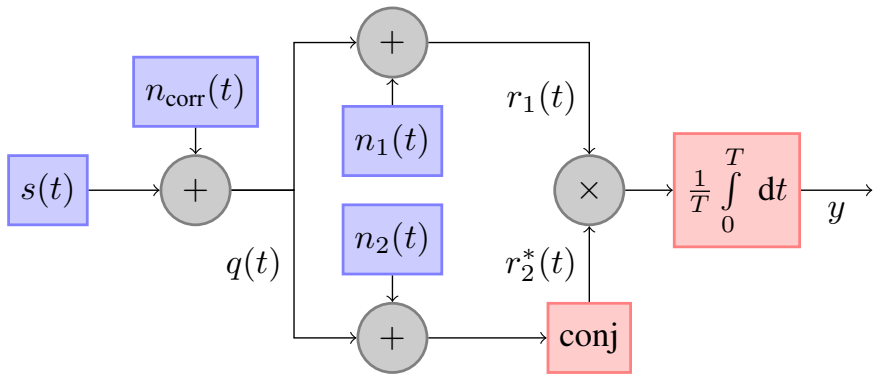

Fig. 4. System model for crosscorrelation.

of $s(\mathrm{t})$ and $n_{\text {corr }}(\mathrm{t})$. The expected output then equals:

$$
\begin{aligned}
\mathbb{E}[y] & =\mathbb{E}\left[r_{1} r_{2}^{*}\right]=\mathbb{E}\left[\left(q+n_{1}\right)\left(q+n_{2}\right)^{*}\right] \\
& =\mathbb{E}\left[|q|^{2}\right]+\mathbb{E}\left[q n_{2}^{*}\right]+\mathbb{E}\left[q^{*} n_{1}\right]+\mathbb{E}\left[n_{1} n_{2}^{*}\right] \\
& =\mathbb{E}\left[|q|^{2}\right]=\mathbb{E}\left[|s|^{2}\right]+\mathbb{E}\left[\left|n_{\text {corr }}\right|^{2}\right]
\end{aligned}
$$

What remains is the autocorrelation or average power of the input signal plus the correlated noise, and thus the Fouriertransform thereof results in the spectrum of only the input signal plus the correlated noise: the uncorrelated noise is removed.

The NF of a system is defined as the deterioration of SNR from input to output. In a crosscorrelation system, the noise contribution of the system is reduced at the cost of measurement time, since $n_{1}$ and $n_{2}$ are averaged out. It can be shown that the effective uncorrelated noise power added by the system decreases with $1.5 \mathrm{~dB}$ for every doubling of measurement time [12]-[14], which can reduce the total system noise by as much as $50 \mathrm{~dB}$ [12]. Hence, one can argue that the SNR of the output increases, or, equivalently, that the $\mathrm{NF}$ of the system goes down. Because this is not the 'standard' definition of NF, we will refer to it as effective $N F$, or in the limit of infinite measurement time, correlated $N F, \mathrm{NF}_{\text {corr }}$.

An important way to increase the SNR for narrowband signals is to reduce the RBW by using more points in the FFT: a doubling of points (doubling of measurement time) halves the noise power per bin, and thus increases the SNR by $3 \mathrm{~dB}$. However, when the bins become smaller than the bandwidth of the signal, both the signal power and the noise power per bin decrease, and the SNR does not improve further. Crosscorrelation can then still improve the SNR by removing the uncorrelated noise, but at a slower rate of $1.5 \mathrm{~dB}$ per doubling of measurement time.

Using crosscorrelation to lower system noise is not a new idea; for instance, it is in widespread use in radio-astronomy [15], and it has been used in [12], [16] to measure the thermal noise of a resistor. In [14], crosscorrelation is discussed (but not implemented) in terms of noise performance, where it is concluded that it "may provide a viable way to relieve the requirement of the analog part of the spectrum sensing receiver." Crosscorrelation was proposed in [17] to mitigate the effects of harmonic downmixing and finite image rejection on spectrum sensing, but the paper does not address noise and linearity issues. 


\section{Crosscorrelation and SNR-wall}

It is well known for energy detection that even the slightest uncertainty in the noise level leads to an SNR-wall: a minimum SNR below which reliable sensing becomes impossible, even with infinite measurement time [18]-[20]. Sonnenschein [21] mentions that crosscorrelation is more robust in the presence of noise uncertainty, and Thompson [15] mentions that it is more robust against gain variations and other analog imperfections. It is shown in [22] that crosscorrelation reduces the SNRwall as compared to autocorrelation, and, close to the SNRwall for autocorrelation, can lower the measurement time by orders of magnitude. These advantages come at the cost of duplication of the frontend and a slight increase in DSP. Note, however, that diversity, MIMO and beamforming systems already have multiple receivers available. Furthermore, when crosscorrelation is not necessary, one of the receivers can simply be turned off, or one can simultaneously use both receivers for autocorrelation, effectively doubling the number of averages in the same amount of time, cutting measurement time in half. Moreover, one could use the two receivers to simultaneously process two different bands.

Apart from the aforementioned differences, crosscorrelation seems to share all properties with autocorrelation. This means that all comparisons between detectors, such as [1], [18], [19], can still be used, while keeping in mind the improved sensitivity of crosscorrelation.

Spectrum sensing techniques capable of extracting signals far below the noise floor are usually computationally intensive and require knowledge of the signals to be detected. The latter aspect is undesired, because the concept of CR applies to any frequency band, where each band may contain a myriad of different modulation techniques. Due to the SNR-wall of energy detection, it is likely that specialized algorithms will still be required in some cases to obtain the desired sensitivity. Therefore, energy detection could be used as a first general method to scan the spectrum and already discard as many occupied bands as possible. As crosscorrelation is a more robust way of energy detection than traditional autocorrelation, it allows specialized algorithms to concentrate on fewer candidate bands.

\section{E. Crosscorrelation and receiver mismatch}

In case of crosscorrelation, the two receivers will have mismatch in their transfer function, affecting the signal power estimate. In essence, the power estimate $\mathcal{P}$ is calculated in the following way (neglecting noise and discrete-time processing):

$$
\mathcal{P}=\left|\int_{\langle\mathrm{BW}\rangle} S(f) \frac{H_{1}(f) H_{2}^{*}(f)}{|H(f)|^{2}} \mathrm{~d} f\right|
$$

where $S(f)$ is the PSD of the signal at the antenna, $H(f)$ is the nominal transfer function of a receiver, $H_{i}$ is the transfer function of receiver $i$, and the integral runs from $f_{0}-\mathrm{RBW} / 2$ to $f_{0}+\mathrm{RBW} / 2$, with $f_{0}$ the center frequency and RBW the bandwidth of the piece of spectrum to be sensed. Note that we take the absolute value, as power must be real and positive.

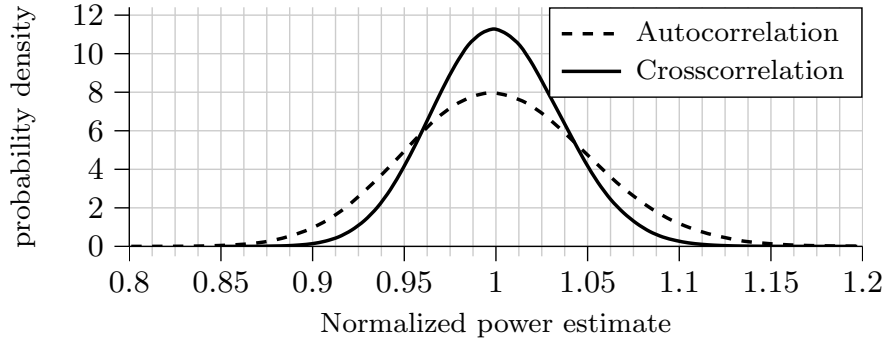

Fig. 5. Simulation results for the power estimation error with $f_{0}=f_{-3 \mathrm{~dB}}=$ $20 \mathrm{MHz}, \mathrm{RBW}=1 \mathrm{MHz}, \sigma_{\epsilon_{i}}=5 \%$ for a single-receiver (autocorrelation) and a two-receiver (crosscorrelation) system. Note that for both functions the integral is 1 , as is required for probability density functions.

To assess the impact of receiver mismatch, let us assume that $S(f)$ is constant over the bandwidth of interest and $H_{1}(f)=H(f)$. To calculate the effect of gain mismatch, we assume $H_{2}(f)=\sqrt{g} H_{1}(f)$ (i.e., receiver 2 has $10 \log _{10} g \mathrm{~dB}$ more gain than receiver 1). It follows from (7) that $\mathcal{P}=$ $P\left|\int_{\langle\mathrm{BW}\rangle} \sqrt{g} \mathrm{~d} f\right|=\sqrt{g} P$. In other words, a gain error of $x \mathrm{~dB}$ in one receiver results in $\frac{x}{2} \mathrm{~dB}$ error in the power estimate. To calculate the effect of phase offset, we assume $H_{2}(f)=e^{j \phi} H_{1}(f)$. We then find $\mathcal{P}=P\left|\int_{\langle\mathrm{BW}\rangle} e^{j \phi} \mathrm{d} f\right|=P$, so a constant phase offset does not influence the power estimate.

When $H(f)$ is a low-pass RC-filter, it gets more complicated as both the gain and the phase mismatch vary across the bandwidth of interest. Let us assume that the $\mathrm{RC}$-product has a Gaussian distribution around its mean

$$
H_{1}=\frac{1}{1+j \omega\left(1+\epsilon_{1}\right) R C} \quad H_{2}=\frac{1}{1+j \omega\left(1+\epsilon_{2}\right) R C}
$$

with $\mu_{\epsilon_{i}}=0$ and $\sigma_{\epsilon_{i}}=0.05$ (i.e., 5\%). One can imagine that the mismatch in the transfer will be worst around the edge of the passband, where gain and phase vary the most. We assume $f_{0}=f_{-3 \mathrm{~dB}}=1 / 2 \pi R C \mathrm{~Hz}=20 \mathrm{MHz}$ and $\mathrm{RBW}=1 \mathrm{MHz}$. Numerically evaluating (7) with the transfer of (8) for random independent $\epsilon_{i}$ yields the pdf of the power estimation error shown in Fig. 5.

Clearly, the crosscorrelator outperforms the autocorrelator in estimation accuracy. This can be intuitively understood by realizing that for autocorrelation (one receiver), $\epsilon_{1}=\epsilon_{2}$, while for crosscorrelation $\epsilon_{1}$ and $\epsilon_{2}$ tend to 'cancel out'. Indeed, simulations indicate that the standard deviation of the power estimation error for crosscorrelation is a factor $\sqrt{2}$ smaller than for autocorrelation.

\section{IMPLEMENTATION}

Traditionally, the trade-off between noise and linearity performance limits the SFDR. The basic philosophy for our implementation is 1) to have a high linearity by design, 2) to improve linearity by attenuation, 3) to reduce the increased noise by crosscorrelation, and 4) to make it suitable for integration on one single CMOS-chip. The block diagram of the system is shown in Fig. 6. We assume that harmonic downmixing in our SA is not the key problem, as it can be reduced by external RF-filters and/or techniques such as 


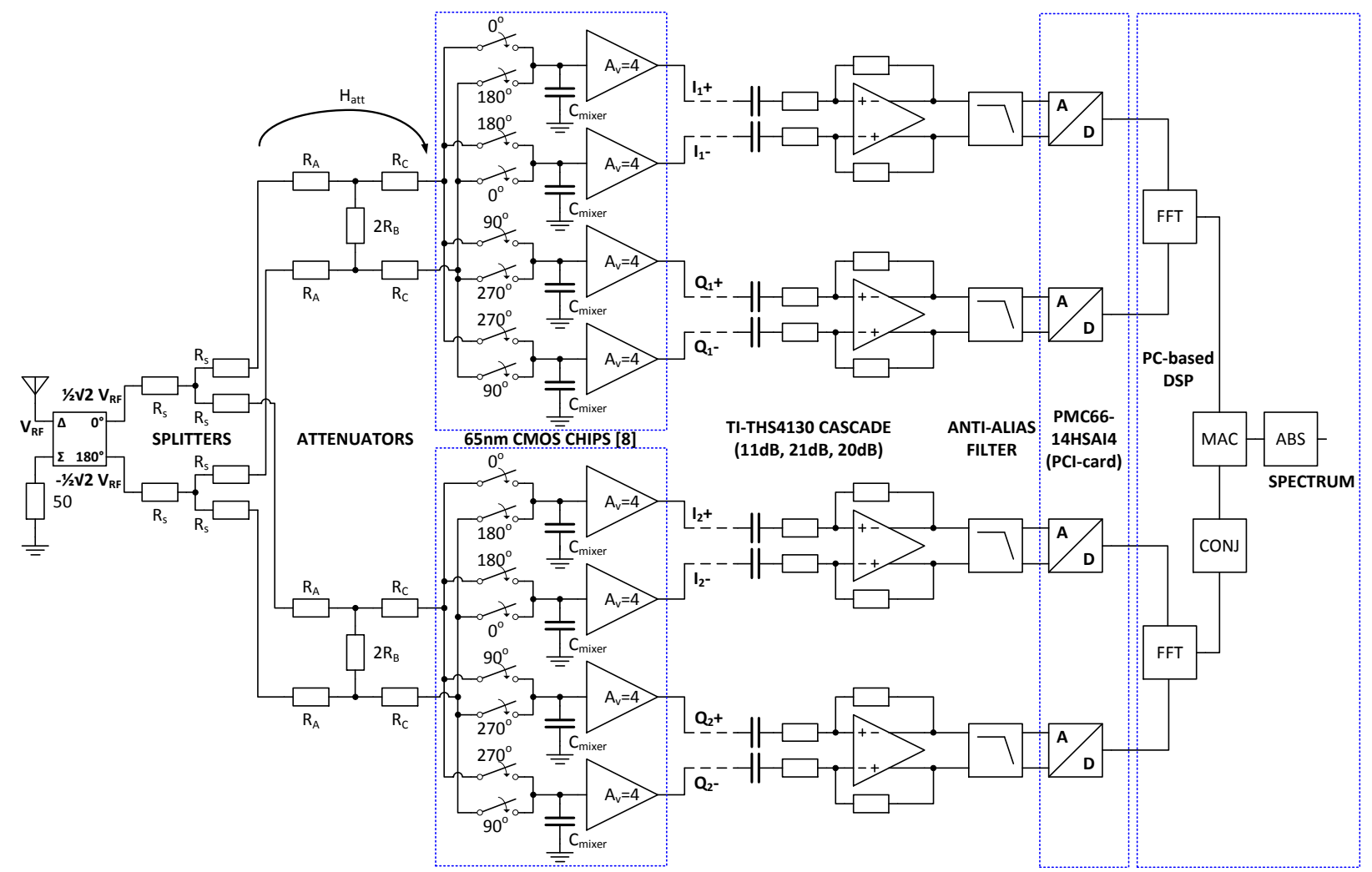

Fig. 6. Implementation of our crosscorrelation system

discussed in [17], [23], and focus on linearity and noise performance.

\section{A. Mixer-first receiver}

The mixer-first architecture of [8] provides a good starting point for high-linearity spectrum analysis. The $65 \mathrm{~nm}$ CMOS chip consists of a mixer and IF-amplifiers, in total achieving IIP3 $=+11 \mathrm{dBm}$ and $\mathrm{NF}=5.5 \mathrm{~dB}$, resulting in $\mathrm{SFDR}=79 \mathrm{~dB}$ in a $1 \mathrm{MHz}$ RBW [8].

The frontend avoids amplification at RF, where linearization techniques such as feedback are inadequate to achieve very high linearity. Instead, the first stage is a passive mixer with only switches and capacitors, which are both very linear in deep-submicron CMOS. Then, at IF, the signal is amplified, where a high loop-gain can be achieved to attain good linearity using resistive feedback. Due to the mixer-first architecture, noise performance is somewhat compromised, but still reasonable at $5.5 \mathrm{~dB}$ NF [8].

The used passive double-balanced quadrature sampling mixer is shown in its single-ended form in Fig. 7 [8]. The switches are controlled by a four-phase non-overlapping square-wave LO with $25 \%$ duty cycle.

The conversion loss of the mixer is $0.9 \mathrm{~dB}$. Due to noise folding, the fundamental minimum DSB NF is $0.9 \mathrm{~dB}$ [8], [24], [25]. The switch-resistance increases this to $1.9 \mathrm{~dB}$. The mixer itself is very linear, with an IIP3 of $+26 \mathrm{dBm}$ [8], [26], but the IF-amplifiers following the mixer limit the linearity to IIP $3=+11 \mathrm{dBm}$. They provide $13.5 \mathrm{~dB}$ voltage gain at a simulated $50 \Omega$-based $\mathrm{NF}$ of $3 \mathrm{~dB}$, which brings the total $\mathrm{NF}$ of the chip to $5.5 \mathrm{~dB}$ [8].

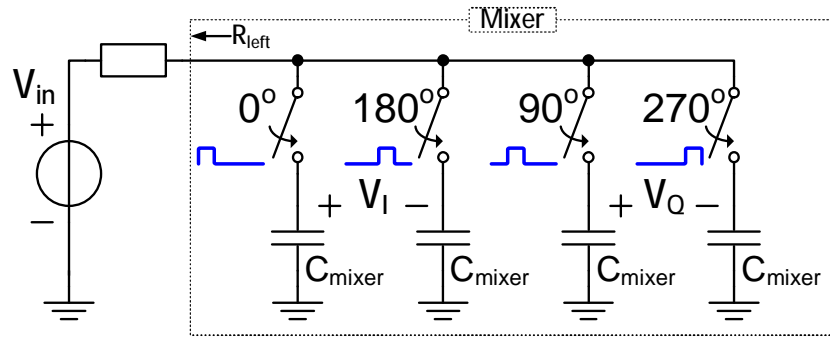

Fig. 7. Schematic of passive quadrature mixer [8].

\section{B. Impedance Matching \& Attenuation}

The input impedance of the passive quadrature mixer in Fig. 7 at the LO-frequency has been analyzed in [24], resulting in the following equation

$$
Z_{\text {mixer }} \approx(X-1) R_{\text {left }}+X R_{\text {switch }}
$$

where $R_{\text {left }}$ is the resistance seen from the mixer looking towards the source, $R_{\text {switch }}$ is the on-resistance of the switches in the mixer, and

$$
X=\left(4 D\left(1-\left(\frac{\sin \pi D}{\pi D}\right)^{2}\right)\right)^{-1}
$$

where $D$ is the duty cycle of the non-overlapping fourphase LO. For our $25 \%$ duty cycle clock this simplifies to $X=\pi^{2} /\left(\pi^{2}-8\right) \approx 5.28$. In a way, we could state that this mixer is "fundamentally" unmatched. For example, for $R_{\text {left }}=50 \Omega$ (a typical RF-source) and $R_{\text {switch }}=5 \Omega$, we find $Z_{\text {mixer }} \approx 240 \Omega$, which is too high to match to $50 \Omega$. It is 
desirable to match to $50 \Omega$, because in our system, as discussed in section II-C, external RF-filters may be required to mitigate harmonic downmixing, and these filters usually require $50 \Omega$ matching.

Besides impedance matching, $R_{\text {left }}$ and $R_{\text {switch }}$ also influence the bandwidth of the mixer at the IF-frequency: together with $C_{\text {mixer }}$ it forms a lowpass $R C$-filter. With a duty cycle of $25 \%$, the resistance seen from the capacitor is effectively quadrupled [8], making the cutoff-frequency

$$
f_{3 \mathrm{~dB}, \mathrm{IF}}=\frac{1}{8 \pi\left(R_{\mathrm{left}}+R_{\mathrm{switch}}\right) C_{\text {mixer }}}
$$

It is possible to provide matching by using baseband components, which, translated by the mixer switches, form an RF-impedance [26]. However, in our case, we can easily combine the functions of impedance matching and attenuation. It is desirable to keep $f_{3 \mathrm{~dB}, \mathrm{IF}}$ equal at different settings of the attenuator, so that the specific setting does not influence the circuitry behind it. Note that the interface between the attenuator and the mixer does not need to be matched (as also exploited in [8]), provided they are located closely enough together.

Overall, we have the following requirements: provide $50 \Omega$ matching, keep $f_{3 \mathrm{~dB}, \mathrm{IF}}$ constant, and provide a certain attenuation $H_{\text {att }}$. Since there are three independent requirements, at least three components are needed to fulfill these requirements, which in Fig. 6 are represented by $R_{A}, R_{B}$, and $R_{C}$. Implementing the attenuator with resistors guarantees wideband operation, high linearity and easy CMOS-integration. Simulations show that an attenuator with discrete settings, implemented with NMOS-switches and resistors, can achieve IIP3 $>+30 \mathrm{dBm}$ in standard $65 \mathrm{~nm}$ CMOS technology for the topology shown in Fig. 6.

Theoretically, the matching network should have a $100 \Omega$ input impedance, such that the parallel application (since we have two paths in parallel) forms a $50 \Omega$ match. However, in our measurement setup, the mixers are physically separated over a significant distance, and the PCB transmission lines have a characteristic impedance of $50 \Omega$. Therefore, we decided to put a resistive power splitter in front, consisting of three resistors with $R_{S}=\frac{R_{\text {src }}}{3} \approx 17 \Omega$, such that each of the matching networks should provide $50 \Omega$ input impedance. This splitter provides a fixed voltage attenuation of $6 \mathrm{~dB}$ from the voltage source to each output of the splitter.

We define $H_{\text {att }}$ without this $6 \mathrm{~dB}$ (see Fig. 6), so

$$
H_{\text {att }}=\frac{Z_{\text {mixer }} R_{B}}{R_{B}\left(Z_{\text {mixer }}+R_{C}\right)+R_{A}\left(Z_{\text {mixer }}+R_{B}+R_{C}\right)}
$$

The input impedance of the system is

$$
Z_{\text {in }}=R_{S}+\frac{1}{2}\left(R_{S}+R_{A}+R_{B} \|\left(R_{C}+Z_{\text {mixer }}\right)\right)
$$

where $x \| y$ means that $x$ and $y$ are in parallel. For $R_{\text {left }}$ we find a long expression, but when input matching is achieved, it simplifies to

$$
R_{\mathrm{left}}=R_{C}+R_{B} \|\left(R_{A}+R_{\mathrm{src}}\right)
$$

With a desired input $Z_{\text {in }}, f_{3 \mathrm{~dB}, \mathrm{IF}}$, and $H_{\text {att }}$, the values of $R_{A}$, $R_{B}$ and $R_{C}$ can be found.
TABLE I

SOME ATTENUATOR COMPONENT VALUES FOR $R_{\mathrm{SRC}}=50 \Omega$, $Z_{\mathrm{IN}}=50 \Omega, f_{3 \mathrm{DB}, \mathrm{IF}}=18 \mathrm{MHz}, C_{\mathrm{MIXER}}=64 \mathrm{PF}$, AND $R_{\mathrm{SWITCH}}=5 \Omega$

\begin{tabular}{cccccc}
\hline$H_{\text {att }}$ & $H_{\text {att }}[\mathrm{dB}]$ & $R_{A}[\Omega]$ & $2 R_{B}[\Omega]$ & $R_{C}[\Omega]$ & used \\
\hline 1.00 & 0 & 0 & 147 & 0 & $\checkmark^{a}$ \\
0.89 & -1 & 5 & 126 & 1 & \\
0.79 & -2 & 10 & 108 & 2 & \\
0.71 & -3 & 14 & 94 & 3 & \\
0.63 & -4 & 17 & 82 & 5 & \\
0.50 & -6 & 24 & 63 & 8 & $\checkmark^{b}$ \\
0.35 & -9 & 31 & 44 & 13 & \\
0.25 & -12 & 36 & 30 & 17 & \\
0.20 & -14 & 39 & 24 & 20 & \\
\hline${ }^{a}$ Refre
\end{tabular}

${ }^{a}$ Referred to as network 1 or $\mathrm{N} 1$

${ }^{b}$ Referred to as network 2 or N2

Table I shows some component values for $Z_{\text {in }}=R_{\text {src }}=$ $50 \Omega, f_{3 \mathrm{~dB}, \mathrm{IF}}=18 \mathrm{MHz}, C_{\text {mixer }}=64 \mathrm{pF}$, and $R_{\text {switch }}=5 \Omega$, which correspond to our design. Note that these values imply $R_{\text {left }}=30 \Omega$. The final column indicates which attenuators are used in the measurements. These attenuator networks will be referred to as network 1 (for $H_{\text {att }}=1.00$ ) and network 2 (for $H_{\text {att }}=0.50$ ) in the remainder of this paper. The choice for these attenuations is based on obtaining a considerable improvement in linearity, while not increasing integration time too much for NF measurements or practical applicability.

\section{Baseband}

A PCI-card with 4 14-bit differential ADCs (PMC6614HSA14) is used to convert the differential IQ-pairs of the receiver outputs to the digital domain, see Fig. 6. The maximum sample rate that could still be handled by the PC operating system was slightly above $11 \mathrm{MSa} / \mathrm{s}$ per channel. The ADCs suffer from a large amount of correlated noise from the many nearby electronics on the board itself and within the $\mathrm{PC}$ (equivalent to roughly $40 \mathrm{~dB} \mathrm{NF}$ ), because they are located inside the PC. It is experimentally found that $10 \mathrm{MSa} / \mathrm{s}$ offers the cleanest spectrum, but still a lot of voltage gain is required to measure the correlated noise from the frontend itself.

This voltage gain is provided by a cascade of low-noise TITHS4130 opamps, in total providing $52 \mathrm{~dB}$ gain $(11 \mathrm{~dB}, 21 \mathrm{~dB}$ and $20 \mathrm{~dB}$, respectively). The high gain causes the DC-offset of the zero-IF receiver $(\approx 7 \mathrm{mV})$ to exceed the input range of the ADCs. We have AC-coupled the final two stages of the opamp cascade with a cutoff frequency of $1 \mathrm{kHz}$, although more sophisticated techniques exist to mitigate DC-offset. These techniques may be applied here as well. The whole cascade of opamps is used for NF-measurements (to overcome the correlated noise of the ADCs), while only the first stage is used for linearity measurements (to prevent the ADCs from saturating). This is also the reason why we cannot directly show the SFDR of the frontend in a single measurement, and we have to derive it from the separate NF and IIP3measurements.

To reduce the noise folding due to sampling at the ADC, a fifth-order passive Butterworth-filter is implemented that 
in combination with the ADCs has a cut-off frequency of $12 \mathrm{MHz}$.

\section{DSP}

In our measurements, we have used 1024-point FFTs to get a good visualization of the spectrum and to be able to filter out the many spurious tones present in our lab environment. To eliminate finite wordlength effects as much as possible, the crosscorrelation is performed on a regular PC in MATLAB using double floating-point precision. Therefore, the digital crosscorrelation is based on an FXC-implementation as discussed in section II-B. Further analysis on finite wordlength effects and fixed-point implementations are left as future work.

The estimated spectrum of the input signal can become complex due to a phase shift introduced by RC-mismatches between the two receivers and timing offsets between the mixers in both receivers [22]. Moreover, the cross-spectrum will be complex with finite measurement time due to the uncorrelated noise injected by the receivers. A possible solution is to take the absolute value after averaging, which is the solution used in this work, see also section II-E. More research is required to find out whether there are better solutions.

\section{EXPECTED SYSTEM PERFORMANCE}

The system performance parameters of interest here are IIP3 and NF, where the NF is interesting before, during and after the crosscorrelation process. IIP3 and NF without crosscorrelation are derived in section IV-A. After removing the uncorrelated noise, crosscorrelation will hit a correlated noise floor. Therefore, we will derive the correlated noise floor of the system in section IV-B, followed in section IV-C by an analysis of the noise floor as a function of measurement time.

\section{A. Performance without crosscorrelation}

The combination of mixer and IF-amplifiers is measured in [8], showing IIP3 $=+11 \mathrm{dBm}$ and $\mathrm{NF}=5.5 \mathrm{~dB}$. Matching provides approximately $3 \mathrm{~dB}$ additional NF (and IIP3) compared to the unmatched case in [8], because we use a passive network to implement the matching. Including the $6 \mathrm{~dB}$ loss of the resistive splitter, the NF and IIP3 will be increased by $9 \mathrm{~dB}$ using network 1 , and $15 \mathrm{~dB}$ using network 2 . The opamps add $2.1 \mathrm{~dB}$ to the NF (calculated using datasheet and feedback resistor values), and the ADCs another $0.7 \mathrm{~dB}$ due to noise folding (calculated using opamp datasheets and measured transfer of anti-alias filter and ADCs). This brings the total for network 1 (network 2) at $\mathrm{NF}=17.3 \mathrm{~dB}(23.3 \mathrm{~dB})$ and IIP3 $=+20 \mathrm{dBm}(+26 \mathrm{dBm})$.

\section{B. Correlated noise floor}

The noise at the output consists of uncorrelated noise power $P_{\mathrm{u}}$ (i.e., noise in a receiver path that has no correlated counterpart in the other receiver path) and correlated noise power $P_{\mathrm{c}}$. For simplicity, we refer these powers to the input and normalize it to the available noise power of the source. In our analysis, we assume no parasitic coupling between the

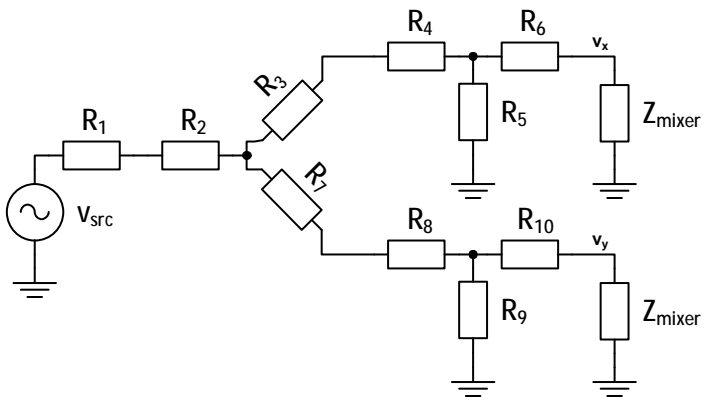

Fig. 8. Schematic for calculating the effect on NF by the splitter plus attenuation network, before and after correlation.

two receivers, and enough gain to make the correlated noise of the ADCs negligible.

In our case, the correlated noise power originates from three contributions:

1) The antenna (or source);

2) The resistors in the splitter and attenuator;

3) Noise folding (of the noise from the previous two effects) in the mixer and the ADCs.

The noise from the antenna/source will be fully correlated in both receivers, limiting the final noise floor to $-174 \mathrm{dBm} / \mathrm{Hz}$. Due to the normalization of $P_{\mathrm{c}}$, we can define $\mathrm{NF}_{\text {corr }}=$ $10 \log _{10} P_{\mathrm{c}}$ as defined in section II-C.

Because of the splitter and the fact that there is no buffer between the attenuator networks in both paths (see Fig. 6), noise generated by these passive components induces a voltage in both paths. Since the crosscorrelator multiplies the voltage outputs of the two receivers (such that the result is already in the power domain), this results in correlated noise power. The noise correlation due to the splitter and attenuators is analyzed using Fig. 8 for the single-ended case. The differential implementation with a balun at the input yields identical results [27].

The antenna noise is modeled by $R_{1}$, the splitter by $R_{2}, R_{3}$ and $R_{7}$, and the attenuation networks by $R_{4}$ to $R_{6}$ and $R_{8}$ to $R_{10}$. It is assumed that the input impedance of the mixer is noiseless. The on-resistance of the switches is not explicitly modeled in this figure, but the generated noise can be treated in the same way as the noise of $R_{6}$ or $R_{10}$; it turns out to have a negligible impact on $\mathrm{NF}_{\text {corr }}$.

The transfer functions $\alpha_{i}$ to node $x$ and $\beta_{i}$ to node $y$ of the noise voltage $v_{n_{i}}$ of each resistor $R_{i}$ can be calculated in a straightforward manner, resulting in instantaneous noise voltages $v_{x}$ and $v_{y}$ of $v_{x}=\sum_{i=1}^{10} \alpha_{i} v_{n_{i}}$ and $v_{y}=\sum_{i=1}^{10} \beta_{i} v_{n_{i}}$. Note that except for $i \in\{1,2,5,9\}, \alpha_{i}$ and $\beta_{i}$ have opposite sign. From symmetry of the circuit it immediately follows for $i \in[3,6]$ that $\alpha_{i}=\beta_{i+4}, \beta_{i}=\alpha_{i+4}$ and $R_{i}=R_{i+4}$.

In our implementation, we have $R_{1}=50 \Omega$ and $R_{2}=R_{3}=$ $R_{7}=17 \Omega$. Using Table I, we have $R_{\text {left }}=30 \Omega$ and thus $Z_{\text {mixer }} \approx 150 \Omega$. For network 2 we then find $\alpha_{1}=0.13, \alpha_{2}=$ $0.13, \alpha_{3}=0.25, \alpha_{4}=0.25, \alpha_{5}=0.59, \alpha_{6}=0.84, \alpha_{7}=$ $-0.13, \alpha_{8}=-0.13, \alpha_{9}=0.11$ and $\alpha_{10}=-0.02$. 
The ccf of $v_{x}$ and $v_{y}$ is

$$
\begin{aligned}
\gamma_{X Y}(\tau) & =\mathbb{E}\left[v_{x}(t) v_{y}^{*}(t+\tau)\right] \\
& =\mathbb{E}\left[\left(\sum_{i=1}^{10} \alpha_{i} v_{n_{i}}(t)\right)\left(\sum_{i=1}^{10} \beta_{i}^{*} v_{n_{i}}^{*}(t+\tau)\right)\right] \\
& =\mathbb{E}\left[\sum_{i=1}^{10} \alpha_{i} \beta_{i}^{*} v_{n_{i}}(t) v_{n_{i}}^{*}(t+\tau)\right] \\
& =\sum_{i=1}^{10} \alpha_{i} \beta_{i}^{*} \mathbb{E}\left[v_{n_{i}}(t) v_{n_{i}}^{*}(t+\tau)\right] \\
& =\sum_{i=1}^{10} \alpha_{i} \beta_{i}^{*} \gamma_{n_{i} n_{i}}(\tau)
\end{aligned}
$$

where the third line follows from the fact that the noise voltage of each resistor is independent of, and, hence, uncorrelated with, the noise voltage of the other resistors. Thus, using linearity of the Fourier transform, the cross-spectrum is

$$
\Gamma_{X Y}(f)=\sum_{i=1}^{10} \alpha_{i} \beta_{i}^{*} \Gamma_{n_{i} n_{i}}(f)
$$

Thus, the correlated noise power from the resistor network can be found from

$$
\begin{aligned}
P_{\mathrm{c}, \mathrm{res}} & =\frac{\sum_{i=1}^{10} \alpha_{i} \beta_{i}^{*} \overline{v_{n, i}^{2}}}{\left|\alpha_{1}\right|^{2} \overline{v_{n, 1}^{2}}} \\
& =\frac{\left|\alpha_{1}\right|^{2} \overline{v_{n, 1}^{2}}+\left|\alpha_{2}\right|^{2} \overline{v_{n, 2}^{2}}+2 \sum_{i=3}^{6} \alpha_{i} \alpha_{i+4}^{*} \overline{v_{n, i}^{2}}}{\left|\alpha_{1}\right|^{2} \overline{v_{n, 1}^{2}}}
\end{aligned}
$$

where $\overline{v_{n, i}^{2}}=4 k T R_{i} \mathrm{~V}^{2} / \mathrm{Hz}$ is the one-sided spectral density of the thermal noise of $R_{i}$ and $\mathrm{NF}_{\text {corr,res }}=10 \log _{10} P_{\mathrm{c}, \text { res }}$. In total, this yields $\mathrm{NF}_{\text {corr,res }}=4.3 \mathrm{~dB}$ for all networks listed in Table I. Simulations using a combination of transient noise in SpectreRF and processing in Matlab verified these results. Thus, for arbitrary attenuation, the NF-reduction will be limited by the splitter and attenuator to a correlated NF of $4.3 \mathrm{~dB}$.

The mixer does not only mix down the signal and noise in the desired frequency band; because it is driven by a squarewave LO, it will also mix down signals and noise present at harmonics of the LO-frequency. Since the paths to both mixer inputs are equal and, to a first approximation, frequencyindependent, the amount of correlation between the noise at both mixer inputs is equal for all frequencies. Hence, noise folding in the mixers increases NF and correlated NF by the same amount, which is $0.9 \mathrm{~dB}$ in our implementation. In total, the derived theoretical minimum NF after crosscorrelation, $\mathrm{NF}_{\text {corr }}$, is $5.2 \mathrm{~dB}\left(\mathrm{~F}_{\text {corr }}=3.3\right)$. Simulations verified these results within $0.7 \mathrm{~dB}$; the frequency translation of the mixer also influences its input in a complicated way, which was not taken into account in (17).

Noise folding also occurs in the ADCs (which adds another estimated $0.7 \mathrm{~dB}$ to the $\mathrm{NF}$ ), but due to the frequency-selective operation of the mixer and the limited bandwidth of the IFcircuitry, the noise folding of correlated noise is expected to be negligible. In other words, noise folding at the ADCs is expected to contribute only to the $\mathrm{NF}$, but not to $\mathrm{NF}_{\text {corr }}$.

\section{Noise floor and measurement time}

With crosscorrelation, the effective NF is lowered as a function of measurement time. To obtain expressions independent of different FFT sizes and sample rates, we introduce the term normalized measurement time (NMT), where $1 \mathrm{NMT}$ is the time required to acquire enough samples for 1 FFT. With our implementation, the expected value of the absolute value of the averaged crosscorrelation output $(\mathbb{E}[|y|])$ is the noise power, with $y$ as defined in Fig. 4.

The uncorrelated noise will be reduced by longer measurement time, but the correlated noise will remain. $P_{\text {init }}=P_{\mathrm{c}}+P_{\mathrm{u}}$ is the input-referred output noise power of a single receiver path, and thus $\mathrm{F}=P_{\text {init }}$, with $\mathrm{F}$ the noise factor of a single path. We define $P_{\mathrm{NMT}}$ as the effective output noise power after averaging NMT FFTs. With finite measurement time, $P_{\mathrm{NMT}}$ will be somewhere in between $P_{\text {init }}$ and $P_{\mathrm{c}}$. An approximate expression was derived in [13], which, applied to our situation, is equal to:

$$
P_{\mathrm{NMT}}=\sqrt{P_{\mathrm{c}}^{2}+\frac{\xi}{\mathrm{NMT}}\left(2 P_{\mathrm{c}} P_{\mathrm{u}}+P_{\mathrm{u}}^{2}\right)}
$$

where $\xi$ is an interpolation function defined as

$$
\xi=\frac{\pi}{4 \mathrm{NMT}}\left(\frac{\Xi\left(\mathrm{NMT}+\frac{1}{2}\right)}{\Xi(\mathrm{NMT})}\right)^{2}\left(1-\frac{P_{\mathrm{c}}^{2}}{\eta}\right)+\frac{1}{2} \frac{P_{\mathrm{c}}^{2}}{\eta}
$$

with $\Xi(x)$ the mathematical Gamma-function, written like this to avoid confusion with the spectrum $\Gamma$, and

$$
\eta=\frac{\mathrm{NMT}+1}{\mathrm{NMT}} P_{\mathrm{c}}^{2}+\frac{1}{\mathrm{NMT}}\left(2 P_{\mathrm{c}} P_{\mathrm{u}}+P_{\mathrm{u}}^{2}\right)
$$

Indeed, $P_{\mathrm{NMT} \rightarrow \infty}=P_{\mathrm{c}}$. Note that when $P_{\mathrm{u}} \gg P_{\mathrm{c}}, P_{\mathrm{NMT}=1} \approx$ $\frac{\pi}{4} P_{\text {init }}$, or equivalently, $\mathrm{NF}_{\mathrm{NMT}=1} \approx \mathrm{NF}-1.05 \mathrm{~dB}$. Since the expressions are a function of NMT, a higher frequency resolution (i.e., more samples per FFT) requires a longer measurement time to obtain the same noise floor (in $\mathrm{dBm} / \mathrm{Hz}$ ).

In section IV-A, we already found that $\mathrm{NF}=17.3 \mathrm{~dB}$ for network 1 and $\mathrm{NF}=23.3 \mathrm{~dB}$ for network 2 , and in section IV-B we found that $\mathrm{NF}_{\text {corr }}=5.2 \mathrm{~dB}$ for both networks. Using (1) and (18), we can derive a general formula for the SFDR of our system as a function of measurement time and $H_{\text {att }}$ :

$$
\mathrm{SFDR}=\frac{2}{3}\left(\mathrm{IIP} 3-\mathrm{NF}_{\mathrm{NMT}}-10 \log _{10} B+174\right)[\mathrm{dB}]
$$

where

$$
\begin{aligned}
\mathrm{IIP} 3 & =\mathrm{IIP} 3_{\mathrm{rcv}}+6-20 \log _{10} H_{\mathrm{att}}[\mathrm{dBm}] \\
\mathrm{NF}_{\mathrm{NMT}} & =10 \log _{10} P_{\mathrm{NMT}}[\mathrm{dB}] \\
P_{\mathrm{c}} & =3.3 \quad P_{\mathrm{u}}=\frac{2 \mathrm{~F}_{\mathrm{rcv}}}{H_{\mathrm{att}}^{2}}-P_{\mathrm{c}}
\end{aligned}
$$

where $P_{\text {NMT }}$ is defined in (18), $H_{\text {att }} \leq 1$ and IIP $3_{\text {rcv }}$ and $\mathrm{NF}_{\text {rcv }}$ denote the IIP3 and NF, respectively, of a single receiver including baseband circuitry and ADCs (see section IV-A), but without attenuator and splitter, which are IIP $3_{\mathrm{rcv}}=+11 \mathrm{dBm}$ and $\mathrm{NF}_{\mathrm{rcv}}=5.5+2.1+0.7=8.3 \mathrm{~dB}$, respectively. 


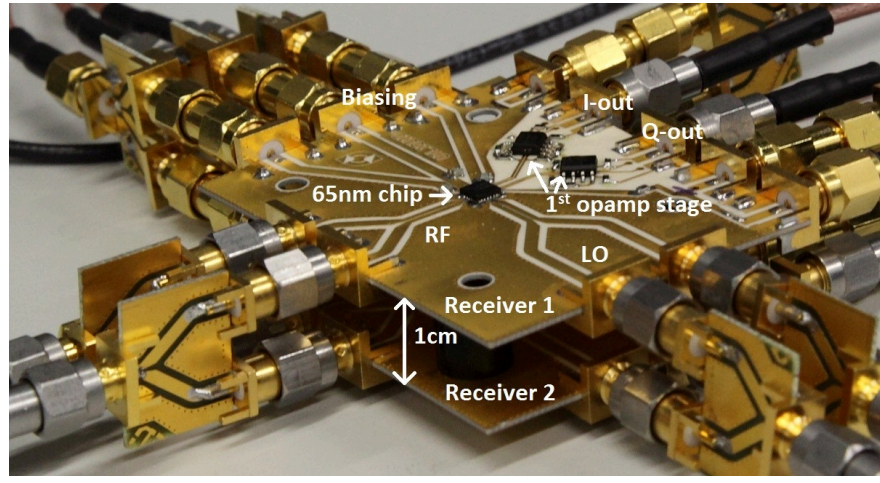

Fig. 9. The measurement setup used, with the two receivers identically oriented with about $1 \mathrm{~cm}$ spacing.

\section{Measurements}

Using the measurement setup as shown in Fig. 9, several measurements were performed. Impedance matching is verified in section V-A for different configurations. Measurement results for the reduction of noise are described in section V-B. In section $\mathrm{V}-\mathrm{C}$ it is shown that crosscorrelation can reduce oscillator phase noise as well. Section V-D summarizes the system performance.

The highest frequency we could measure was at $1.5 \mathrm{GHz}$ due to the measurement setup, while the mixer can operate up to $2.0 \mathrm{GHz}$, only limited by the clocking circuitry. The lowest frequency for which the mixer still works is around $50 \mathrm{MHz}$, while in [8] it was only measured down to $200 \mathrm{MHz}$ to demonstrate a decade of RF bandwidth. Also, we measured the mixer to have a $\mathrm{NF}=5.5 \mathrm{~dB}$ over the whole band, as opposed to the $6.5 \mathrm{~dB}$ as reported in [8].

\section{A. Impedance Matching}

Matching is important, because the system itself does not provide adequate filtering, and requires an RF-filter at the input. Because of the unusual input impedance of the mixer [see (9)], $S_{11}$ is measured for several situations. Fig. 10a shows the measured and simulated $S_{11}$ of a single mixer plus network 1 for one specific $f_{\mathrm{LO}}(200 \mathrm{MHz})$. Fig. 10b shows the measured $S_{11}$ of the same configuration for many different $f_{\mathrm{LO}}$, where, for clarity, only the measurement results around $f_{\text {LO }}$ are shown. These results clearly show that there is good matching around $f_{\mathrm{LO}}$.

Fig. 10c shows the measured results for two different $f_{\mathrm{LO}}$, for several different situations (only mixer (M), mixer and attenuation network $(\mathrm{M}+\mathrm{N} 1, \mathrm{M}+\mathrm{N} 2)$, and splitter with two parallel mixers and attenuation networks $(2 \mathrm{M}+\mathrm{S}+\mathrm{N} 1,2 \mathrm{M}+\mathrm{S}+\mathrm{N} 2))$. This picture shows that the mixer itself is unmatched, and that the matching/attenuation networks improve matching. The large dip around $1.1 \mathrm{GHz}$ for splitter + network $2+$ two mixers is probably a PCB or cable effect, as it is present for arbitrary $f_{\mathrm{LO}}$. It is interesting to note that for $f_{\mathrm{LO}}=1400 \mathrm{MHz}$, the matching peaks differ in frequency for the two networks with splitter and two mixers in parallel. This effect is also present for $f_{\mathrm{LO}}=300 \mathrm{MHz}$, but much less pronounced. Simulations indicate that this effect may be caused by parasitic capacitances in front of the mixer (bondpad, PCB), which also explains why it is more pronounced at higher frequencies.

\section{B. Noise Figure}

An output spectrum for different measurement times is shown in Fig. 11, where it can be seen that the noise floor is lowered for longer measurement time. Some peaks are visible in the spectrum, which may originate from PCs, measurement equipment, supplies or other nearby labs. They were discarded in the determination of the noise floor level. Eventually, the noise floor remains at a certain level, which is the residual correlated noise. The spectra with low NMT look smoother because they have been averaged for better visibility of the trend in noise reduction. Without this smoothing, the variance on a dB-scale would be the same for all NMT until the noise floor gets close to the correlated noise floor.

For these 1024-point spectral estimates at a sample rate of $10 \mathrm{MSa} / \mathrm{s}$, a NMT of 1 is equal to $0.1 \mathrm{~ms}$ to obtain a RBW of $10 \mathrm{kHz}$. With a maximum NMT of $2^{15}$, it took $3.3 \mathrm{~s}$ to acquire enough samples. The higher noise around the center frequency is caused by DC-offset and flicker noise of the final opamp stage and ADCs (the DC-offset and flicker noise of the CMOS chips are filtered out by the AC-coupling).

The theoretical effective NF as a function of measurement time for network 1 is plotted in Fig. 12, together with some representative measurement results. The theoretical $\mathrm{NF}$ is based on (18), using $\mathrm{NF}=17.3 \mathrm{~dB}$ and $\mathrm{NF}_{\text {corr }}=5.2 \mathrm{~dB}$, as discussed in section IV. The measurement results are based on the noise floor such as shown in Fig. 11, with a test tone to determine the total gain of the system. Clearly, the measured correlated NF is sometimes significantly lower and sometimes significantly higher than the theoretically predicted $5.2 \mathrm{~dB}$. For network 2, similar results are obtained, with roughly the same correlated NF at the same frequency.

The deviation between $\mathrm{NF}_{\text {corr }}$ as derived in section IV-B and the measured correlated noise floor as shown in Fig. 12 may be explained by several factors:

- the transfers calculated using Fig. 8 assume no time delay between the noise from a resistor arriving at $v_{x}$ and $v_{y}$, while in our setup the time difference for some resistors is $0.50 \mathrm{~ns}$ due the length of the PCB transmission lines, which changes the values of $\alpha_{i}$ in (17);

- the mixers perform frequency translation, which affects their inputs, resulting in much more complicated relations between $v_{x}$ and $v_{y}$ due to the different phase-shifts of each frequency component;

- the two receivers were stacked on top of each other, such that the transmission lines on the PCB ran in parallel, isolated only by a centimeter of air (see Fig. 9), which introduces additional frequency-dependent coupling;

- interference from outside can introduce positive or negative correlation when it couples to the two receivers, depending on the phase difference.

We intend to explore these effects in more detail in future work.

We have fitted the two parameters $P_{\mathrm{c}}$ and $P_{\mathrm{u}}$ in (18) to match the measured effective NF for different RF-frequencies 


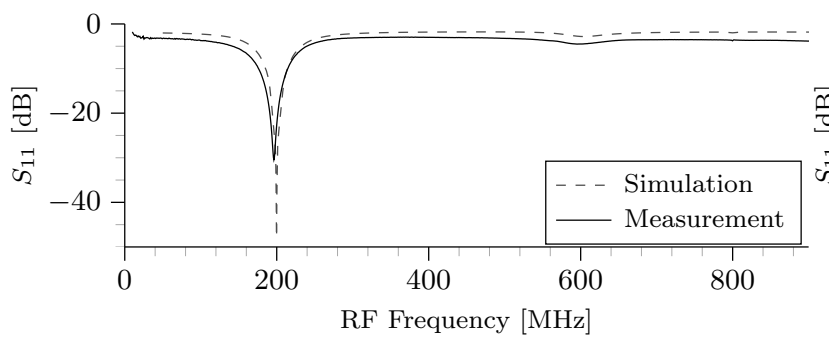

(a) Single mixer with network 1 with $f_{\mathrm{LO}}=200 \mathrm{MHz}$

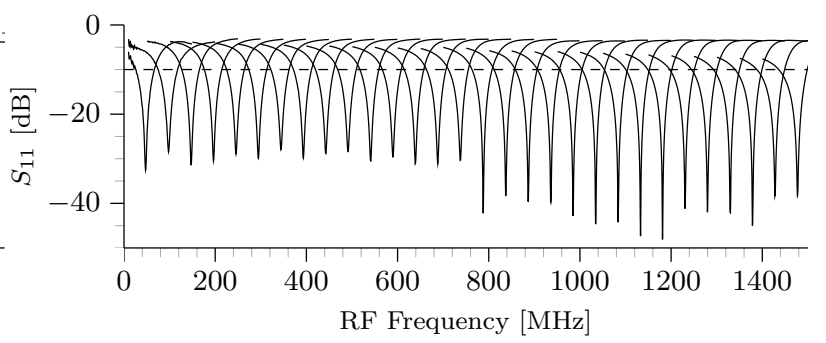

(b) Single mixer with network 1 with $f_{\mathrm{LO}}=k \cdot 50 \mathrm{MHz}$

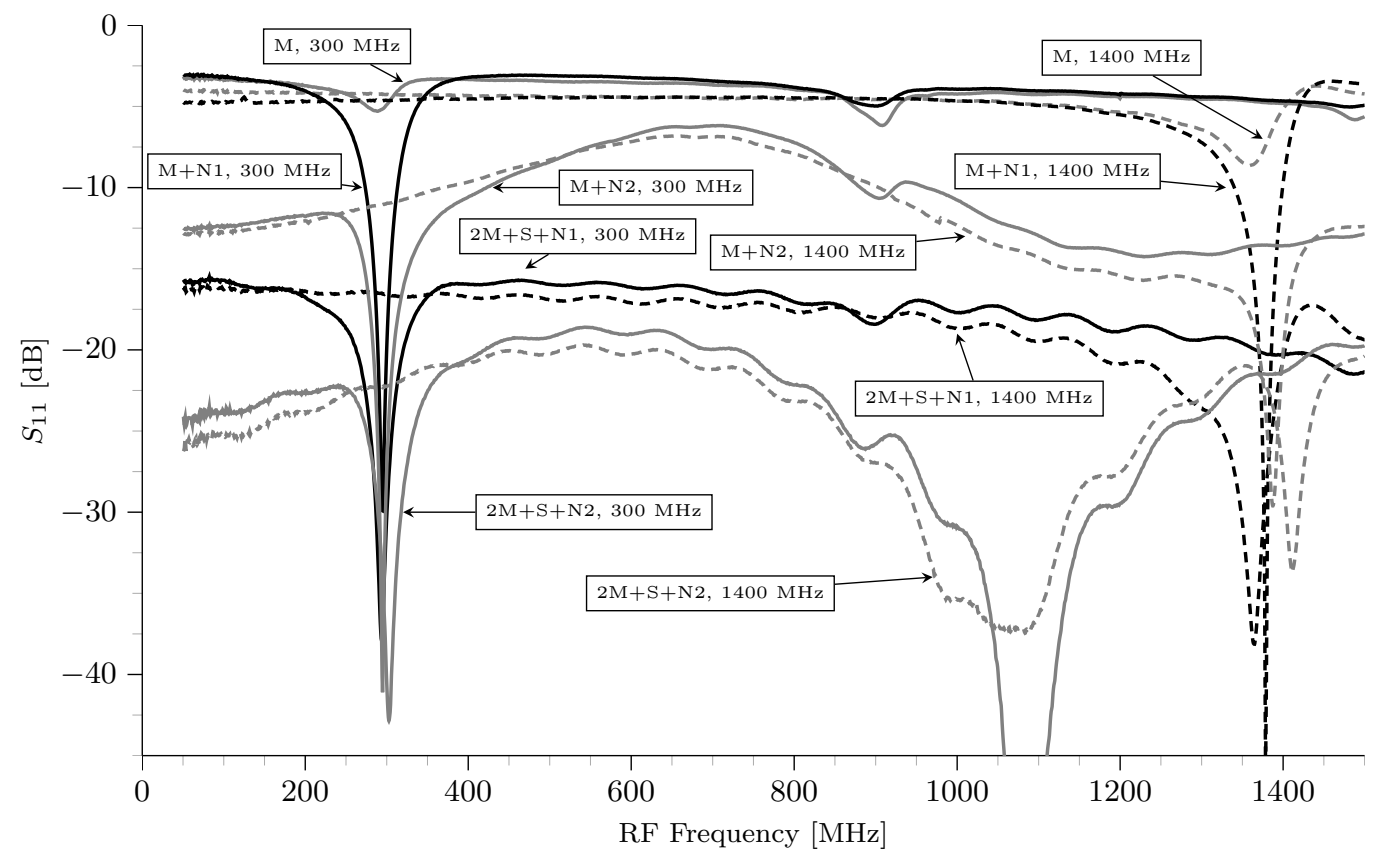

(c) Measurement results for different situations with $f_{\mathrm{LO}}=300 \mathrm{MHz}$ and $f_{\mathrm{LO}}=1400 \mathrm{MHz}$. M denotes mixer, N1 denotes network 1, N2 denotes network 2, and $\mathrm{S}$ denotes the splitter.

Fig. 10. Measurement results for $S_{11}$.

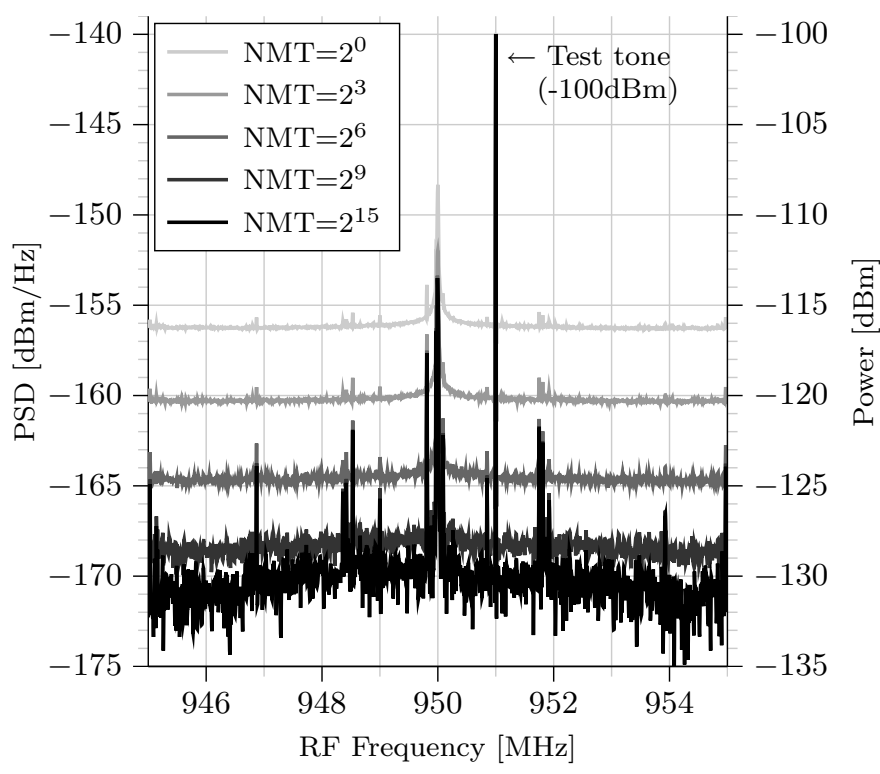

Fig. 11. Measured spectrum as function of correlation time. The test tone of $-100 \mathrm{dBm}$ was inserted to verify correct operation. The RBW in this measurement is $10 \mathrm{kHz}$. using network 1, see Fig. 12. Using only these two parameters, the match with the measurements is excellent for any NMT (for $400 \mathrm{MHz}$ : $\mathrm{NF}=17.2 \mathrm{~dB}, \mathrm{NF}_{\text {corr }}=9.3 \mathrm{~dB}$; for $600 \mathrm{MHz}$ : $\mathrm{NF}=16.8 \mathrm{~dB}, \mathrm{NF}_{\text {corr }}=1.8 \mathrm{~dB}$; for $860 \mathrm{MHz}: \mathrm{NF}=15.3 \mathrm{~dB}$, $\left.\mathrm{NF}_{\text {corr }}=5.7 \mathrm{~dB}\right)$. We obtain similar agreement with network 2 (not shown). Hence, we believe that our measurements are performed correctly, and that the aforementioned list explains the differences that sometimes exist between calculated and measured $\mathrm{NF}_{\text {corr }}$.

When using the frontend with network 2, ADCs sampling at $10 \mathrm{MSa} / \mathrm{s}$, and a RBW of $1 \mathrm{MHz}$, obtaining enough samples for each FFT takes $1 \mu \mathrm{s}$. From (18), the effective NF decreases from $24 \mathrm{~dB}$ to below $10 \mathrm{~dB}$ after 900 FFTs, which takes only $0.9 \mathrm{~ms}$. For CR this measurement time can be acceptable, as IEEE 802.22 requires a channel detection time of $2 \mathrm{~s}$ [28], and both FCC (USA) and Office of Communications (UK) require a sensing interval in the order of a second [2], [29]. Note that standard energy detection also requires averaging to reduce the variance to achieve reasonable performance for relatively weak signals. 


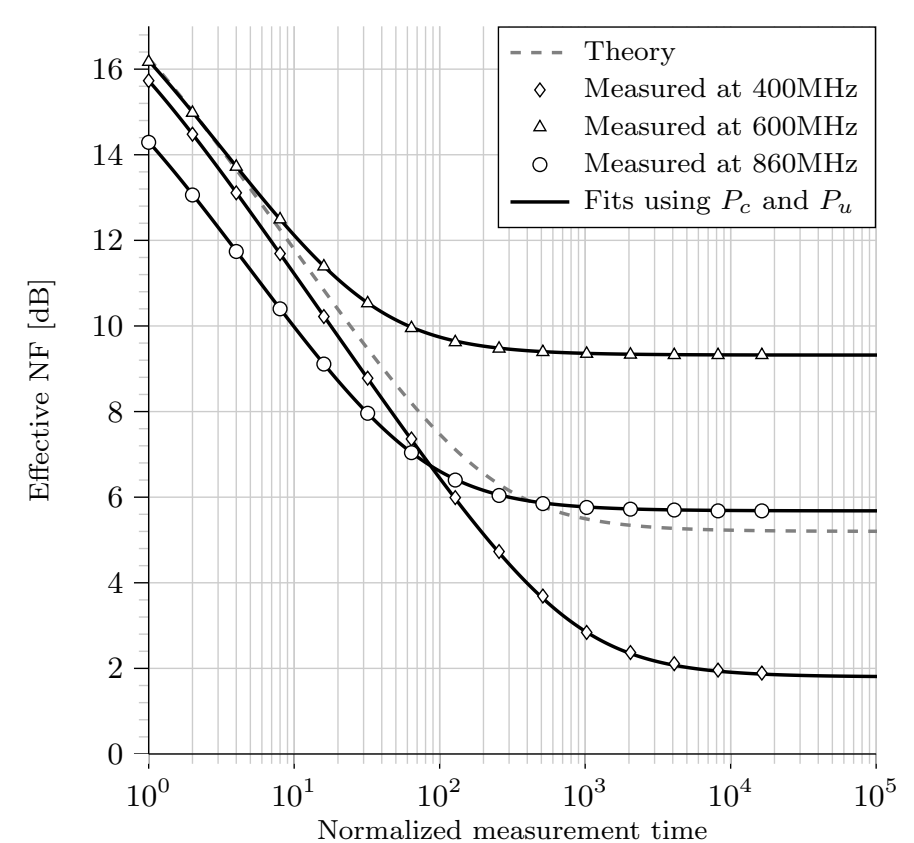

Fig. 12. Theory (dashed) using section IV-C with $\mathrm{NF}=17.3 \mathrm{~dB}$ and $\mathrm{NF}_{\text {corr }}=5.2 \mathrm{~dB}$, measured effective NF (markers) and theoretical fits (black) using (18) as a function of measurement time for attenuation network 1.

\section{Phase Noise Reduction}

One of the other factors that can limit SFDR is phase noise. Phase noise manifests itself mostly around strong input signals [30], thereby obscuring weak signals in their vicinity. Crosscorrelation offers a unique opportunity to reduce phase noise by employing two separate oscillators, as is also done in some professional phase noise analysis devices [31]. These oscillators need to be frequency-locked, and, depending on the architecture, phase-locked. Their phase noise will be uncorrelated, apart from the phase noise of a common (external) reference, which can be very low and thus negligible.

Because in our system the oscillators are external and have good phase noise performance, phase noise is emulated by applying PM-modulation. The modulation is done by independent white noise sources, such that the phase noise of both oscillators is independent. The synthesizers are frequencylocked using one of the two as reference for the other, while the phases are synchronized manually before the start of each experiment. Each mixer is then driven by one of the oscillators. The phase synchronization is necessary, because otherwise the impedance seen by one mixer at its input is affected by the other mixer.

The obtained spectra with attenuation network 2 are shown in Fig. 13. The autocorrelation spectra are the spectra as observed at the output of each single receiver. Because the LO-signal generators are of different brands, it is likely that the PM-modulation input is handled in a different way, which explains why the spectra of the two receivers differ. This is, however, not a key concern for demonstrating the principle of phase noise reduction. For crosscorrelation with NMT=1, the observed spectrum is roughly halfway (in $\mathrm{dBs}$ ) between the autocorrelation spectra. Clearly, a longer crosscorrelation measurement then reduces the phase noise.

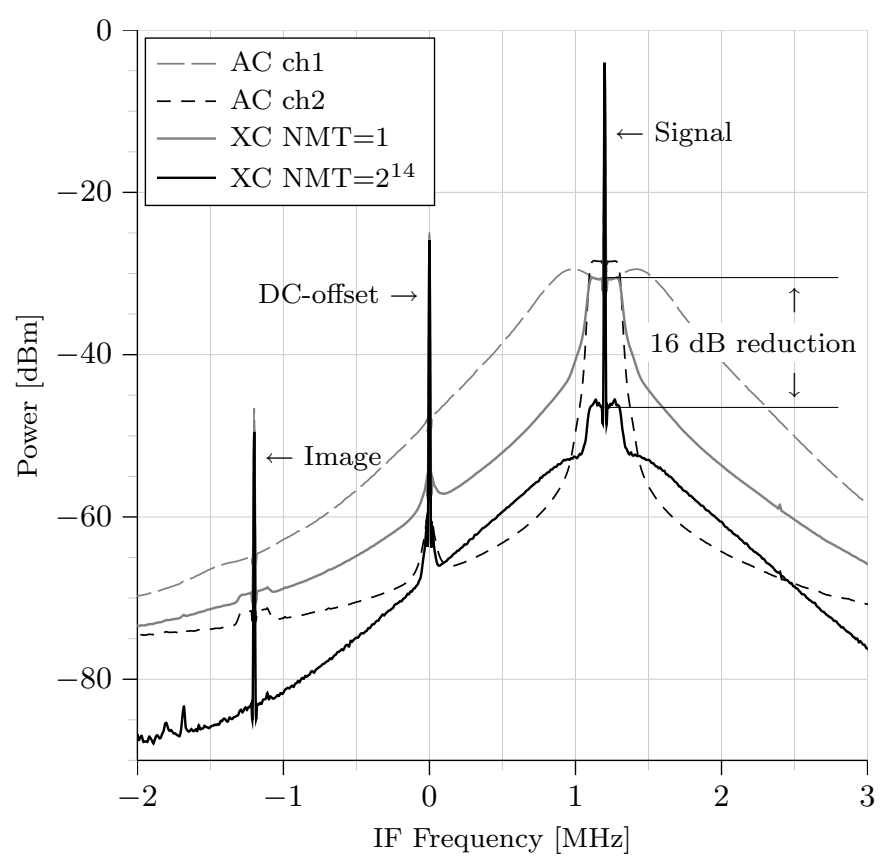

Fig. 13. Measurement results for phase noise reduction using two PMmodulated frequency-locked oscillators with attenuation network 2. AC denotes autocorrelation, XC crosscorrelation.

It can be observed that there is some crosstalk between the two receivers, as the phase noise is reduced by $16 \mathrm{~dB}$, and not to negligible levels. This is confirmed by an experiment where only one of the two oscillators is modulated and phase noise showed up in the other path as well (not shown). We expect this coupling to scale with the attenuation: $1 \mathrm{~dB}$ additional attenuation provides an additional $2 \mathrm{~dB}$ isolation between the mixers, and thus $1 \mathrm{~dB}$ reduction in the correlated phase noise. Hence, we expect $10 \mathrm{~dB}$ phase noise reduction with attenuation network 1 , which is close to the measured $11 \mathrm{~dB}$ (not shown).

\section{Overall performance}

The NF and IIP3 are measured over the entire bandwidth of operation (with a common low phase-noise oscillator that, in contrast with the previous subsection, is not modulated), of which the results are shown in Fig. 14. The measured NF corresponds with the predicted NF $(17.3 \mathrm{~dB}$ and $23.3 \mathrm{~dB}$, see section IV-A) $\pm 1.5 \mathrm{~dB}$, while the measured IIP3 is about $1.5 \mathrm{~dB}$ lower than calculated in section IV $(20 \mathrm{dBm}$ and $26 \mathrm{dBm})$.

It can be clearly seen that the additional $6 \mathrm{~dB}$ of attenuation increases both NF and IIP3 by $6 \mathrm{~dB}$, but the additional noise is completely reduced by crosscorrelation. This is in accordance with the calculations of section IV, showing that the lower bound on NF after crosscorrelation is independent of the attenuation. As a result, using (1), the $6 \mathrm{~dB}$ of attenuation increases SFDR by $4 \mathrm{~dB}$. For both attenuation networks, the measured correlated NF fluctuates between $1 \mathrm{~dB}$ and $11 \mathrm{~dB}$, which is on average roughly equal to the NF of the original frontend without attenuator. For network 1, the SFDR is (on average) increased by $5 \mathrm{~dB}$ as compared to the single frontend, while for network 2 the improvement on average is $9 \mathrm{~dB}$. 
TABLE II

COMPARISON WITH OTHER DESIGNS

\begin{tabular}{|c|c|c|c|c|c|c|c|c|c|}
\hline Architecture & Technology & $\begin{array}{c}\text { RF Freq. } \\
{[\mathrm{GHz}]}\end{array}$ & $\begin{array}{l}\text { Power } \\
{[\mathrm{mW}]}\end{array}$ & VSWR & $\begin{array}{l}\mathrm{NF}_{1} \\
{[\mathrm{~dB}]}\end{array}$ & $\begin{array}{l}\mathrm{NF}_{2} \\
{[\mathrm{~dB}]}\end{array}$ & $\begin{array}{l}\text { Time } \\
\text { penalty } \\
\text { (NMT) }\end{array}$ & $\begin{array}{c}\text { IIP3 } \\
{[\mathrm{dBm}]}\end{array}$ & $\begin{array}{c}\text { SFDR } \\
(\mathrm{RBW}=1 \mathrm{MHz}) \\
{[\mathrm{dB}]}\end{array}$ \\
\hline This work (network 1) & $65 \mathrm{~nm}$ CMOS & $0.05-1.5$ & $191^{a}$ & $<1.2$ & 17 & 5 & $273^{b}$ & 17 & $83^{c}$ \\
\hline This work (network 2) & $65 \mathrm{~nm}$ CMOS & $0.05-1.5$ & $191^{a}$ & $<1.2$ & 23 & 5 & $4333^{b}$ & 24 & $88^{c}$ \\
\hline Soer et al. [8] & $65 \mathrm{~nm}$ CMOS & $0.2-2.0$ & 67 & $4.8^{d}$ & 5.5 & & & 11 & 79 \\
\hline Park et al. [32] & $0.18 \mu \mathrm{m} \mathrm{CMOS}$ & $0.4-0.9$ & 180 & & $50^{e}$ & & & $-17^{f}$ & 31 \\
\hline Kitsunezuka et al. [33] & 90nm CMOS & $0.03-2.4$ & 37 & & $39^{e}$ & & & -11 & 42 \\
\hline Pollin/Ingels et al. [34], [35] & 40nm CMOS & $0.1-6.0$ & $100^{g}$ & & 3 & & & -12 & 66 \\
\hline Tektronix RSA2203A & & $0-3$ & & $<1.4$ & 24 & & & 30 & $70 / 80^{h}$ \\
\hline Agilent PXA N9030A-503 & & $0-3.6$ & & $<1.2$ & 18 & $8^{i}$ & $200^{j}$ & 22 & 85 \\
\hline
\end{tabular}

${ }^{a}$ Only includes clock-dividers, switch drivers, integrated IF-amplifiers and estimated DSP power consumption

${ }^{b}$ Calculated NMT to have an effective NF within $1 \mathrm{~dB}$ of $\mathrm{NF}_{\text {corr }}$

${ }^{c}$ Using the value $\mathrm{NF}_{\text {corr }}+1$ as $\mathrm{NF}$

${ }^{d}$ Calculated at mixing frequency using [24]

${ }^{e}$ Calculated based on sensitivity given in $\mathrm{dBm}$ in a certain bandwidth

${ }^{f} \mathrm{P}_{1 \mathrm{~dB}}$ given in paper as $-27 \mathrm{dBm}$

${ }^{g}$ Analog frontend + 10-bit ADCs

${ }^{h}$ Datasheet (including phase noise) / calculated using IIP3 and NF

${ }^{i}$ Using noise floor extension (which estimates noise power at start-up and subtracts it from measurement result)

${ }^{j}$ Calculated NMT based on doubled variance due to subtraction, and a $10 \mathrm{~dB}$ lower expected value

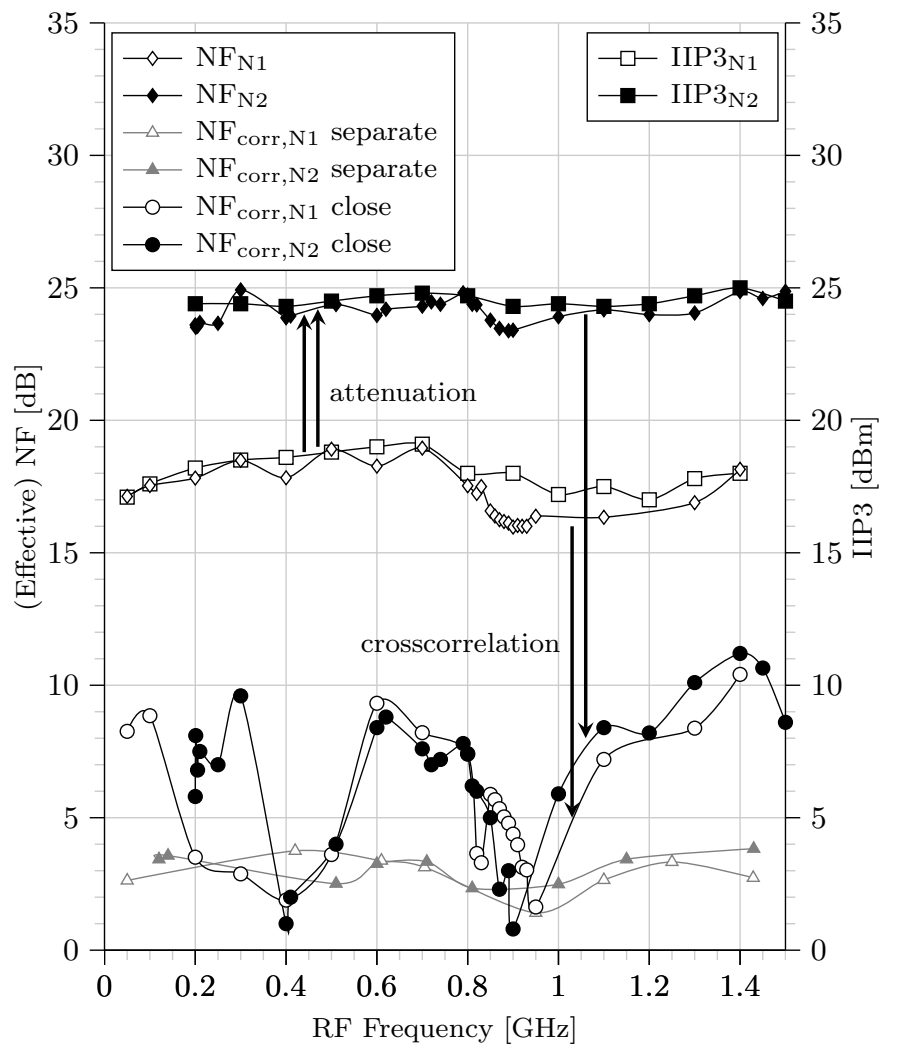

Fig. 14. NF and IIP3 measurement results. Here, "separated" denotes the situation where the two receivers were physically separated by about $50 \mathrm{~cm}$, while "close" denotes the situation where the two receivers are physically very close, as shown in Fig. 9.

A note on these measurements as compared to the measurements we published in [9] is in place here. In [9], the PCBs were physically separated, and we found a noise floor that was flat over most frequencies. For reference, these measurements are also shown in Fig. 14. In this paper, we made new measurements with the two receivers much closer together, see Fig. 9. For the case of separated boards, the noise floor after crosscorrelation is much lower for many frequencies. The achievable noise floor for crosscorrelation is limited by correlated noise, and crosstalk between the receivers will introduce correlated noise. However, this experimental demonstrator is likely to have different crosstalk mechanisms from an integrated spectrum analyzer, which is what we aim for. Therefore we decided to report both measurement results in two extreme cases: board in close proximity and separated. Further work on an integrated version has to show how much isolation can practically be achieved.

Each analog frontend (mixer + IF-amplifier) consumes $61 \mathrm{~mW}\left(f_{\mathrm{LO}}=50 \mathrm{MHz}\right)$ to $83 \mathrm{~mW}\left(f_{\mathrm{LO}}=1.5 \mathrm{GHz}\right)$. The DSP-part consists of FFTs and MACs. To compare the power consumption in the integrated analog part with that of the digital part, if it were integrated, we assume a fixed-point implementation. To accommodate the high SFDR using fixedpoint numbers, we assume 24-bit FFTs (a simulation using 16-bit fixed-point 1024-point FFTs showed a maximum SFDR of $87 \mathrm{~dB}$ after crosscorrelation) and enough additional bits at the accumulators to allow for long integration times. Using the figures of [36] and scaling from 8-bit to 24-bit $(\times 9)$, from $2.4 \mathrm{GSa} / \mathrm{s}$ to $20 \mathrm{MSa} / \mathrm{s}(\div 120)$, from 1 channel to 2 channels $(\times 2)$, and allowing $10 \%$ more for the MACs, we estimate the DSP power consumption at $25 \mathrm{~mW}$ when it is integrated on chip. The analog power consumption is relatively high, but the crosscorrelation concept can be used with other frontends as well. In this respect, the total power consumption will be maximally twice as high as that of an ordinary receiver, depending on how many components can be shared (e.g., the oscillator when phase noise is not restricting).

Table II compares the results to several other architectures, showing the high linearity and low noise of our design.

\section{CONCLUSIONS}

Spectrum sensing for CR requires very strong and very weak signals to be measured at the same time. Deep- 
submicron CMOS-processes are a good candidate for CRapplications because of the low cost and the DSP-capabilities required in such devices. Unfortunately, especially linearity becomes a problem for the low supply voltages inherent to modern CMOS-processes. Passive attenuation improves linearity, but degrades noise performance, which prevents weak signals from being detected. This trade-off can be summarized in the SFDR, which is limited by thermal noise, phase noise, and non-linearity.

In this paper it is shown that duplication of the frontend and crosscorrelation of the outputs of the two frontends can significantly reduce the noise. It relies on the principle that the noise introduced by each frontend is (mostly) uncorrelated. Theoretically, each doubling of measurement time reduces the uncorrelated parts of the system-induced noise by $1.5 \mathrm{~dB}$, which is verified by measurements. This concept allows passive attenuation to improve linearity, while the loss in noise performance is made up by crosscorrelation. It is also shown that the crosscorrelation technique can considerably reduce phase noise, which could otherwise negatively affect SFDR.

The residual noise level is calculated to be $5.2 \mathrm{~dB}$, which is verified by simulations. However, the measurements show relatively large fluctuations (several $\mathrm{dBs}$ ) around this value, which cannot be explained by measurement errors alone, and requires more research. Nevertheless, a significant reduction in noise level, and, hence, improvement in SFDR, is obtained within acceptable measurement time. The achieved IIP3 of $+24 \mathrm{dBm}$ is $13 \mathrm{~dB}$ better than what is achieved using a single frontend, while the final noise floor is similar. In total, the SFDR is increased from $79 \mathrm{~dB}$ to $88 \mathrm{~dB}$ in a $1 \mathrm{MHz} \mathrm{RBW}$, which compares favorably to other designs.

Therefore, crosscorrelation enables integrated SAs in CMOS with high SFDR and sensitivity, making it a good candidate for spectrum sensing for $\mathrm{CR}$, or for spectral analysis in general.

\section{ACKNOWLEDGEMENTS}

We thank A.R. Smeenge, N.A. Moseley, M.J. Bentum, A. Ghaffari and M. Heskamp for many fruitful discussions, G.J.M. Wienk and H. de Vries for the practical assistance and NXP for providing silicon. This research is supported by the Dutch Technology Foundation STW, applied science division of NWO and the Technology Program of the Ministry of Economic Affairs (project 08081).

\section{REFERENCES}

[1] R. Tandra, A. Sahai, and S. M. Mishra, "What is a spectrum hole and what does it take to recognize one?" Proc. IEEE, vol. 97, no. 5, pp. 824-848, 2009.

[2] FCC, "In the matter of unlicensed operation in the TV broadcast bands and additional spectrum for unlicensed devices below $900 \mathrm{MHz}$ and in the 3 GHz band," FCC, Tech. Rep., Nov. 2008.

[3] D. H. Mahrof, E. A. M. Klumperink, J. C. Haartsen, and B. Nauta, "On the effect of spectral location of interferers on linearity requirements for wideband cognitive radio receivers," in Proc. 4th IEEE Symp. on New Frontiers in Dynamic Spectrum Access Networks (DySPAN), Apr. 6-9 2010, pp. 1-9.

[4] P. F. Marshall, "Dynamic spectrum management of front end linearity and dynamic range," in Proc. 3rd IEEE Symp. on New Frontiers in Dynamic Spectrum Access Networks (DySPAN), 2008, pp. 1-12.
[5] T. H. Lee, The Design of CMOS Radio-Frequency Integrated Circuits. Cambridge University Press, 2004.

[6] C. Rauscher, Fundamentals of Spectrum Analysis, 1st ed. Rohde \& Schwarz, 2001.

[7] B. Razavi, RF Microelectronics. Prentice Hall, 1998.

[8] M. C. M. Soer, E. A. M. Klumperink, Z. Ru, F. E. van Vliet, and B. Nauta, "A 0.2-to-2.0GHz 65nm CMOS receiver without LNA achieving $>11 \mathrm{dBm}$ IIP3 and $<6.5 \mathrm{~dB}$ NF," in Proc. IEEE Int. Solid-State Circuits Conf. - Dig. Tech. Papers, 2009, pp. 222-223,223a.

[9] M. S. Oude Alink, E. A. M. Klumperink, M. C. M. Soer, A. B. J. Kokkeler, and B. Nauta, "A 50MHz-to-1.5GHz cross-correlation CMOS spectrum analyzer for cognitive radio with $89 \mathrm{~dB}$ SFDR in $1 \mathrm{MHz} \mathrm{RBW}$," in Proc. 4th IEEE Symp. on New Frontiers in Dynamic Spectrum Access Networks (DySPAN), 6-9 Apr. 2010, pp. 1-6.

[10] J. Proakis, C. Rader, F. Ling, C. Nikias, M. Moonen, and I. Proudler, Algorithms for Statistical Signal Processing. Prentice Hall, 2002.

[11] J. D. Bunton, "SKA correlator advances," Experimental Astronomy, vol. 17, no. 1-3, pp. 251-259, Jun. 2004.

[12] M. Sampietro, G. Accomando, L. G. Fasoli, G. Ferrari, and E. Gatti, "High sensitivity noise measurement with a correlation spectrum analyzer," IEEE Trans. Instrum. Meas., vol. 49, no. 4, pp. 820-822, Aug. 2000 .

[13] J. Briaire and L. K. J. Vandamme, "Uncertainty in gaussian noise generalized for cross-correlation spectra," J. Appl. Phys., vol. 84, pp. 4370-4374, 1998.

[14] M. Heskamp and C. Slump, "Sub-noise primary user detection by crosscorrelation," in Proc. Int. Conf. on Communications (ICC), Jun. 2009.

[15] A. Thompson, J. Moran, and J. G.W. Swenson, Interferometry and Synthesis in Radio Astronomy, 4th ed. Krieger Publishing Company, 1998.

[16] C. Ciofi, F. Crupi, and C. Pace, "A new method for high-sensitivity noise measurements," IEEE Trans. Instrum. Meas., vol. 51, no. 4, pp. 656-659, 2002.

[17] N. A. Moseley, E. A. M. Klumperink, and B. Nauta, "A spectrum sensing technique for cognitive radios in the presence of harmonic images," in Proc. 3rd IEEE Symp. on New Frontiers in Dynamic Spectrum Access Networks (DySPAN), 2008, pp. 1-10.

[18] D. Cabric, A. Tkachenko, and R. W. Brodersen, "Spectrum sensing measurements of pilot, energy, and collaborative detection," in Proc. IEEE Military Communications Conference (MILCOM), 2006, pp. 1-7.

[19] R. Tandra and A. Sahai, "SNR walls for feature detectors," in Proc. 2nd IEEE Symp. on New Frontiers in Dynamic Spectrum Access Networks (DySPAN), 2007, pp. 559-570.

[20] — "Noise calibration, delay coherence and SNR walls for signal detection," in Proc. 3rd IEEE Symp. on New Frontiers in Dynamic Spectrum Access Networks (DySPAN), 2008, pp. 1-11.

[21] A. Sonnenschein and P. M. Fishman, "Radiometric detection of spreadspectrum signals in noise of uncertain power," IEEE Trans. Aerosp. Electron. Syst., vol. 28, no. 3, pp. 654-660, 1992.

[22] M. S. Oude Alink, A. B. J. Kokkeler, E. A. M. Klumperink, G. J. M. Smit, and B. Nauta, "Lowering the SNR-wall for energy detection using crosscorrelation," IEEE Trans. Veh. Technol. (accepted).

[23] Z. Ru, N. Moseley, E. Klumperink, and B. Nauta, "Digitally enhanced software-defined radio receiver robust to out-of-band interference," IEEE J. Solid-State Circuits, vol. 44, no. 12, pp. $3359-3375$, dec. 2009.

[24] B. W. Cook, A. Berny, A. Molnar, S. Lanzisera, and K. S. J. Pister, "Low-power 2.4-GHz transceiver with passive RX front-end and 400mV supply," IEEE J. Solid-State Circuits, vol. 41, no. 12, pp. 2757-2766, 2006.

[25] M. C. M. Soer, E. A. M. Klumperink, P.-T. de Boer, F. E. van Vliet, and B. Nauta, "Unified frequency-domain analysis of switched-seriespassive mixers and samplers," IEEE Trans. Circuits Syst. I, vol. 57, no. 10 , pp. 2618-2631, Oct. 2010.

[26] C. Andrews and A. C. Molnar, "A passive-mixer-first receiver with baseband-controlled RF impedance matching, $<6 \mathrm{~dB} \mathrm{NF}$, and $>27 \mathrm{dBm}$ wideband IIP3," in Proc. IEEE Int. Solid-State Circuits Conf. - Dig. Tech. Papers, Feb. 2010, pp. 46-47.

[27] A. A. Abidi and J. C. Leete, "De-embedding the noise figure of differential amplifiers," IEEE J. Solid-State Circuits, vol. 34, no. 6, pp. 882-885, 1999.

[28] C. Stevenson, G. Chouinard, Z. Lei, W. Hu, S. Shellhammer, and W. Caldwell, "IEEE 802.22: The first cognitive radio wireless regional area network standard," IEEE Commun. Mag., vol. 47, no. 1, pp. 130$138,2009$.

[29] Office of Communications, "Statement on licence-exempting cognitive devices using interleaved spectrum," Office of Communications, Statement, Jul. 2009. 
[30] V. J. Arkesteijn, E. A. M. Klumperink, and B. Nauta, "Jitter requirements of the sampling clock in software radio receivers," IEEE Trans. Circuits Syst. II, vol. 53, no. 2, pp. 90-94, 2006.

[31] Rohde \& Schwarz, "FSUP Quick Start Guide," Jan. 2008

[32] J. Park, T. Song, J. Hur, S. M. Lee, J. Choi, K. Kim, K. Lim, C.H. Lee, H. Kim, and J. Laskar, "A fully integrated UHF-band CMOS receiver with Multi-Resolution Spectrum Sensing (MRSS) functionality for IEEE 802.22 cognitive radio applications," IEEE J. Solid-State Circuits, vol. 44, no. 1, pp. 258-268, 2009.

[33] M. Kitsunezuka, H. Kodama, N. Oshima, K. Kunihiro, T. Maeda, and M. Fukaishi, "A $30 \mathrm{MHz}-2.4 \mathrm{GHz}$ CMOS receiver with integrated RF filter and dynamic-range-scalable energy detector for cognitive radio," in Proc. IEEE Radio Frequency Integrated Circuits Symp. (RFIC), 2011, pp. 1-4.

[34] S. Pollin, E. Lopez, A. Antoun, P. v. Wesemael, L. Hollevoet, A. Bourdoux, A. Dejonghe, and L. v. d. Perre, "Digital and analog solution for low-power multi-band sensing," in Proc. 4th IEEE Symp. on New Frontiers in Dynamic Spectrum Access Networks (DySPAN), 2010.

[35] M. Ingels, V. Giannini, J. Borremans, G. Mandal, B. Debaillie, P. Van Wesemael, T. Sano, T. Yamamoto, D. Hauspie, J. Van Driessche, and J. Craninckx, "A 5mm2 40nm LP CMOS 0.1-to-3GHz multistandard transceiver," Proc. IEEE Int. Solid-State Circuits Conf. - Dig. Tech Papers, pp. 458-459, feb. 2010.

[36] Y. Chen, Y.-W. Lin, Y.-C. Tsao, and C.-Y. Lee, "A 2.4-Gsample/s DVFS FFT processor for MIMO OFDM communication systems," IEEE $J$ Solid-State Circuits, vol. 43, no. 5, pp. 1260-1273, 2008.

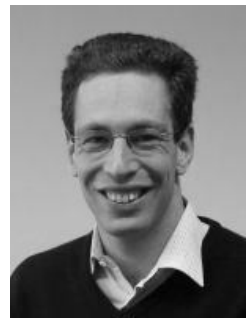

Mark S. Oude Alink (S'09) was born on June 20, 1984, in Hengelo, the Netherlands. He received the B.Sc. degree in computer science in 2004 and the M.Sc. degrees in electrical engineering and computer science (both cum laude) in 2008, all from the University of Twente (UT), Enschede, the Netherlands.

$\mathrm{He}$ is currently working towards the $\mathrm{Ph} . \mathrm{D}$. degree at the IC-Design group and the Computer Architecture for Embedded Systems group at the UT. His research includes system design, spectrum sensing and modulation for cognitive radio.

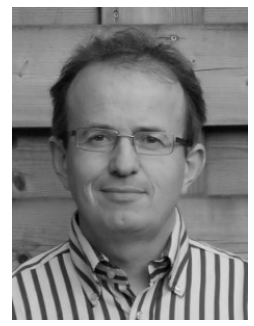

Eric A. M. Klumperink (M'98-SM'06) was born on April 4, 1960, in Lichtenvoorde, the Netherlands. He received the B.Sc. degree from HTS, Enschede, the Netherlands, in 1982. After a short period in industry, he joined the Faculty of Electrical Engineering of the University of Twente (UT) in Enschede in 1984, participating in analog CMOS circuit design and research. This resulted in several publications and a Ph.D. thesis in 1997 ("Transconductance based CMOS circuits")

After receiving his Ph.D., he started working on RF CMOS circuits. He is currently an Associate Professor at the IC-Design Laboratory which participates in the CTIT Research Institute (UT). He holds several patents and has authored or coauthored more than 80 journal and conference papers.

In 2006 and 2007, Dr. Klumperink served as an Associate Editor for IEEE Transactions on Circuits and Systems II, and since 2008 for IEEE Transaction on Circuits and Systems I. He was a corecipient of the ISSCC 2002 Van Vessem Outstanding Paper Award.

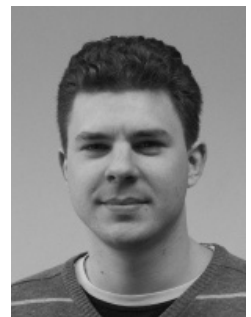

Michiel C. M. Soer (S'09) was born in Schoonhoven, the Netherlands, in 1984 . He received the M.Sc. degree (cum laude) in electrical engineering from the University of Twente, Enschede, the Netherlands, in 2007

He is currently working towards the Ph.D. degree at the IC-Design group at the same university. His research interests include mixers, discrete time systems and phased arrays in CMOS.

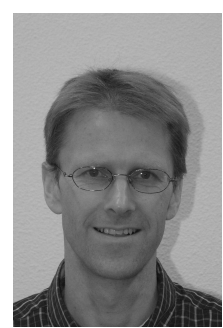

André B. J. Kokkeler has worked more than 6 years at Ericsson as a system engineer and 8 years at the Netherlands foundation for research in astronomy (ASTRON) as a scientific project manager. In 2003 he joined the University of Twente. In 2005 he obtained his $\mathrm{Ph} . \mathrm{D}$. degree on the thesis "Analogdigital Codesign using Coarse Quantization". He has a background in telecommunication, mixedsignal design and signal processing. Currently, his main interest lies in the area of applying low-power design techniques for computationally intensive applications. The emphasis is on reconfigurable architectures for streaming applications. He is involved in research projects, sponsored by the Dutch and European governments and industry.

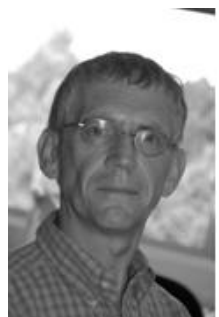

Gerard J. M. Smit received his M.Sc. degree in electrical engineering from the University of Twente, Enschede, The Netherlands. He finished his Ph.D. thesis entitled "The Design of Central Switch Communication Systems for Multimedia Applications" in 1994. He currently is a Full Professor with the faculty of EEMCS, University of Twente, leading the CAES chair, where he is responsible for a number of research projects sponsored by the EC, industry and Dutch government in the field of multimedia and efficient reconfigurable systems.

After receiving the M.Sc. degree, he worked for four years at the Research laboratory of Océ, Venlo, the Netherlands. In 1994, he was a Visiting Researcher with the Computer Laboratory, Cambridge University, Cambridge, UK and, in 1998, he was a Visiting Researcher with Lucent Technologies Bell Labs Innovations, Murray Hill, NJ. Since 1999, he has been leading the Chameleon group, which investigates new hardware and software architectures for energy-efficient systems. Currently his research interests include lowpower communication, and reconfigurable architectures for energy reduction.

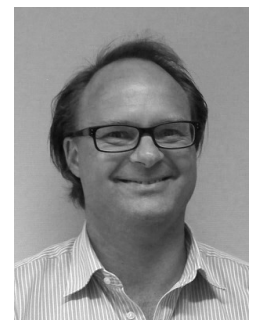

Bram Nauta (M'91-SM'03-F'08) was born in Hengelo, the Netherlands. In 1987, he received the M.Sc. degree (cum laude) in electrical engineering from the University of Twente, Enschede, the Netherlands. In 1991, he received the Ph.D. degree from the same university on the subject of analog CMOS filters for very high frequencies. In 1991, he joined the MixedSignal Circuits and Systems Department of Philips Research, Eindhoven, the Netherlands. In 1998, he returned to the University of Twente as full Professor heading the IC Design group. His current research interest is high-speed analog CMOS circuits.

$\mathrm{He}$ served as Associate Editor of IEEE Transactions on Circuits and Systems-II (1997-1999). He was Associate Editor (2001-2006) and later the Editor-in-Chief (2007-2010) of IEEE Journal of Solid-State Circuits. He is member of the technical program committees of the International SolidState Circuits Conference (ISSCC), where he is the 2012 Program Committee vice-Chair, the European Solid-State Circuit Conference (ESSCIRC), and the Symposium on VLSI Circuits. He is co-recipient of the ISSCC 2002 and 2009 "Van Vessem Outstanding Paper Award", is distinguished lecturer of the IEEE, elected member of IEEE-SSCS AdCom and is IEEE fellow. 